REVISTA

MEXICANA DE

ECONOMÍA Y

FINANZAS

REMEF

(TIE MEXICAN JOURNAL OF

ECONOMICS AND FINANCE
Revista Mexicana de Economía y Finanzas, Nueva Época

Volumen 17 Número 2, Abril - Junio 2022, pp. 1-32, e531

DOI: https://doi.org/10.21919/remef.v17i2.531

(Primera recepción: 31/julio/2020, última recepción: 29/julio/2021 aceptado: 31/agosto/2021, publicado: 5/noviembre/2021)

\title{
Estimación bayesiana del modelo de difusión con saltos de Merton
}

\author{
Miguel Antonio Alba Suarez ${ }^{1}$ - Universidad Santo Tomás, Colombia \\ Miguel Ángel Alba Acosta - Universität Potsdam, Alemania \\ David Camilo Alba Acosta - Universidad Santo Tomás, Colombia
}

En la literatura existen diferentes aportes en la forma como se puede identificar la evolución de los derivados financieros vía precios de los activos subyacentes. El Modelo de Difusión con Saltos de Merton (MDSM) es una de las referencias más importantes para modelar la dinámica estocástica de los rendimientos de los activos en comparación con el modelo de Black y Scholes (B\&S). El objetivo principal de este trabajo es realizar un análisis comparativo entre el MDSM y el B\&S desde un enfoque bayesiano utilizando métodos Markov-Chain-Monte-Carlo (MCMC).

Las simulaciones aplicadas al registro diario de algunas de las principales acciones que conforman el índice NASDAQ evidenciaron la superioridad en ajuste del MDSM sobre los rendimientos financieros vía MCMC. Algunas recomendaciones y limitaciones de esta investigación surgen en la propuesta adecuada para los valores usados como parámetros para las distribuciones a priori previas a la estimación de las distribuciones posterior para cada parámetro de cada modelo.

El mayor aporte dentro del marco estadístico de esta investigación es ilustrar la efectividad los métodos MCMC para MDSM en yuxtaposición a B\&S.

Clasificación JEL: B23, C15, C53, F37, G13.

Palabras clave: Modelo de Difusión con saltos de Merton (MDSM), estadística bayesiana, MCMC, Modelo de Black \& Scholes (B\&S), procesos estocásticos.

\section{On the Bayesian Estimation of Merton's Jump Diffusion Model}

In the literature there are different contributions on how to identify the evolution of financial derivatives via underlying asset prices. The Jump-difussion-Merton's model (JDMM) is one of the most important references to model the stochastic dynamics of asset returns in comparison with the Black and Scholes (B\&S) model. The main objective of this paper is to perform a comparative analysis between the JDMM and the B\&S from a Bayesian approach using Markov-Chain-Monte-Carlo (MCMC) methods.

Simulations applied to the daily log of some of the main stocks that make up the NASDAQ index evidenced the superiority in goodness of fit of the JDMM over financial returns via MCMC. Some recommendations and limitations of this research arise in the appropriate proposal for the values used as parameters for the prior distributions used before estimating the posterior distributions for each parameter of each model.

The major contribution within the statistical framework of this research is illustrating the effectiveness of the MCMC methods for the JDMM in juxtaposition to B\&S.

JEL Classification: C61, C63, G12, G17, G14.

Keywords: Merton's jump diffusion model (JDMM), Bayesian statistics, MCMC, Black \& Scholes (B\&S) model, stochastic processes.

${ }_{1}^{1}$ Autor de correspondencia. Email: miguelalba@usantotomas.edu.co

Facultad de Negocios Internacionales, Universidad Santo Tomás, Colombia

*Sin fuente de financiamiento para el desarrollo de la investigación 


\section{Introducción}

En el mundo de los derivados financieros, uno de los productos más demandados en el mercado son las opciones, que tienen la característica en ser contratos que dan el derecho a su propietario de comprar o vender un activo denominado subyacente en un tiempo determinado.

En el ejercicio de valorar las opciones, la literatura ha contado con diferentes modelos entre los que se destacan: B\&S (1973), Heston (1993), Duan (1995), Binomial (1979) y el modelo de Difusión con saltos de Merton (1975).

B\&S es uno de los modelos más utilizados por el mercado; sin embargo, este se caracteriza por ser un proceso determinista, es decir, no tiene la particularidad de explicar el precio de las opciones a explosiones abruptas generadas por una volatilidad ininterrumpida. Ante esta situación, surge el modelo Merton, el cual se caracteriza en explicitar los cambios abruptos denominados "saltos" que se pueden presentar en un momento determinado, originado o causado por la volatilidad vía escenarios de comportamiento mixto vías caídas y subidas repentinas. Usualmente este modelo descrito es usado, cuando los rendimientos subyacentes son discontinuos (Merton, 1976); y por esta razón, es también es considerado en la literatura y en el mercado, como un instrumento para resolver problemas cuando se presentan "colas pesadas" en las distribuciones de los rendimientos.

En general, los modelos de difusión con saltos han sido una herramienta con gran aplicabilidad por parte del sistema financiero; en estos modelos, el proceso que siguen los precios resulta ser un movimiento browniano, y la probabilidad de movimientos en las acciones en grandes intervalos en el corto plazo resulta ser pequeña, debido a que los precios a corto plazo en las opciones

"out of the money" son mucho más pequeños de lo que se observa en los mercados. Asimismo, si los precios de las opciones en acciones se les permite un salto incluso cuando el vencimiento de estas se da en el corto plazo, existe una probabilidad que después de este cambio las opciones estén "in the money".

En cuanto a las operaciones de derivados, se pueden incluir uno o más instrumentos con el fin de realizar operaciones de cubrimiento en todo el mercado; sin embargo, es necesario identificar que la presencia de saltos en los precios en el mercado no permite un perfecto cubrimiento, y las opciones permiten de alguna manera a los agentes cubrirse frente a situaciones adversas mitigando el riesgo, que no puede ser cubierto con un solo activo subyacente (Venegas-Martínez, 2008).

Desde una perspectiva de la gestión de riesgo, se ha corroborado que el uso de modelos de saltos es importante, ya que, su uso permite observar el riesgo del precio de los movimientos accionarios en pequeños intervalos de tiempo, los cuales no son previsibles en la llamada difusión (Tankov \& Voltchkova, 1992); por lo tanto, teniendo en cuenta el método de preservación del capital (CPPI) se observa que, al tomar desde el punto de vista matemático una proporción $x_{t}$ del riesgo del activo en el portafolio como $x_{t}=m \frac{V_{t}-F_{t}}{V_{t}}$, siendo $V_{t}$ el valor del portafolio, $F_{t}$ el valor mínimo que puede tener este $m$ un multiplicador, si el valor del portafolio tiende al valor mínimo $F_{t}$ el riesgo se minimiza. En ese sentido, teniendo un modelo continuo, los portafolios nunca tenderán a este valor mínimo representado, y al tener una constante de multiplicación amplia se puede minimizar el riesgo; sin embargo, el riesgo en la mayoría de las situaciones se expresa con saltos esporádicos que aumentan la probabilidad que el inversionista ante un cambio brusco vía la tendencia baja para el 
activo no pueda tener la oportunidad de diversificar sus inversiones antes de llegar al valor mínimo de la cartera.

De acuerdo con lo anterior, MDSM en la literatura es considerado como una de las metodologías de estimación más precisa para identificar y representar la dinámica estocástica de los precios cuando se presentan variaciones bruscas en los rendimientos (Castillo, 2009).

Desde el punto de vista de la estimación clásica para MDSM su ejecución resulta como una mixtura de normales producto de la distribución de los rendimientos de los activos. En ese sentido, cuando se examina el mercado que se caracteriza en muchas ocasiones por contar con casos de mayor complejidad, se requiere en la lectura de MDSM un número considerable de saltos lo que dificulta la estimación debido a la función de verosimilitud cuya característica principal es ser multimodal. Bajo esa óptica desde la lectura clásica, la magnitud de los saltos es tratada como una constante en el modelo.

Cabe de igual manera resaltar, que, a pesar de la volatilidad del mercado, así como las restricciones que se pueden generar, las estimaciones de MDSM logran superar las estimaciones de B\&S; no obstante, la estimación bayesiana por medio de los algoritmos de "Markov Chain Monte Carlo (MCMC)" puede ser una solución mediática a estos problemas.

Conforme a lo explicitado, la construcción de los algoritmos de simulación vía Markov Chain Monte Carlo (MCMC) permite generar muestras de una distribución a posteriori a partir de una $a$ priori y una verosimilitud dada. La noción de las cadenas de Markov se fundamenta en que cada valor generado depende del valor simulado inmediatamente anterior (Conchi, 2012), y, por otro lado, Monte Carlo se caracteriza por aproximar expresiones que se dificultan calcular analíticamente recolectando muestras, para el cómputo de aproximaciones, por medio de estimadores empíricos como lo son: la media de los datos.

La ventaja de estas técnicas de estimación descritas es poder expresar el precio final de una opción en términos de la expectativa del precio generado por el modelo sobre las distribuciones de los parámetros. Algunos autores como Gupta y Reisinger (2012) asumen que los modelos financieros pueden ser calibrados mediante la estimación bayesiana para los modelos de valoración de opciones, la cual, la convierte en una herramienta para generar una metodología consistente en la obtención de distribuciones a posteriori de los precios de ellas en el mercado. Asimismo, es necesario circunscribir que el comportamiento de los precios y rendimientos de los activos financieros subyacentes de las opciones se da por la hipótesis del mercado eficiente.

Es importante de igual manera resaltar que, el mercado es afectado por diferentes fundamentales que influyen necesariamente en el precio de los activos, los cuales en muchas ocasiones son externalidades que inciden en el comportamiento numérico de los mismos. Ante esta realidad, el modelamiento matemático y estadístico surge como respuesta para comprender y entender la volatilidad como los saltos, y dar una aproximación de lo que está sucediendo en el mercado con supuestos que simplifiquen las influencias exógenas a las series financieras propiamente dichas. Se puede entender en este contexto, que la aleatoriedad de un activo financiero se debe al comportamiento histórico reflejado en el tiempo presente, y a la respuesta que puede generar el mercado nuevas informaciones sobre el comportamiento del activo.

En general MDSM es una extensión de B\&S; y por lo tanto, se considera que los procesos difusos capturan la volatilidad generada por distintas actividades de trading, y que los saltos capturan los componentes más significativos en los cambios de los precios de los activos debido a 
nueva información; estos saltos generan asimetría y curtosis en los rendimientos de los precios (Tunaru \& Zheng, 2017).

Lo explicitado anteriormente, de alguna manera reafirma que, las propiedades markovianas de las series financieras al no contar con ningún tipo de memoria, permiten la implementación de modelos independientes al comportamiento pasado, y al modelamiento de nuevas informaciones que afecte el precio del activo (Fathaleden Asiri, 2018).

Adicionalmente, el presente trabajo evidencia e ilustra los supuestos y consideraciones sobre la evolución del precio de los activos subyacentes con base en los modelos B\&S y MDSM, utilizando metodologías de carácter bayesiano, así como propuestas sobre las distribuciones a priori asociadas al espacio de parámetros sobre la distribución de los procesos estocásticos vinculados a ambos modelos.

Finalmente, para la realización de los cálculos se utilizó el software R y la base de datos de Bloomberg. Las acciones que fueron objeto de estudio pertenecen al índice accionario NASDAQ, y el marco temporal de estudio fue el 23 de mayo de 2014 al 23 de junio de 2021.

\section{Definición teórica de MDSM}

Para hablar sobre el MDSM es necesario tener en cuenta algunas consideraciones matemáticas para su definición; en ese aspecto, la parte de difusión hace referencia al proceso estocástico de movimiento browniano que siguen los precios de las opciones. En este sentido, un proceso estocástico, es un modelo matemático que permite capturar el comportamiento ininterrumpido en un tiempo de determinado suceso o magnitud aleatoria con determinado espacio muestral.

En general, una sucesión de eventos aleatorios que se desarrolla en un transcurso de tiempo se le denomina proceso estocástico, es decir, la evolución de una variable aleatoria en determinado tiempo, inclusive en lapsos reducidos y de manera continua. La expresión matemática de los eventos aleatorios se puede explicitar de la siguiente manera:

$$
\boldsymbol{X}=\{X(t), t \in T\}
$$

donde $\boldsymbol{X}$ es una colección de variables aleatorias, lo que quiere decir que para cada $t$ en el índice $T$ existe $X(t)$ es una variable aleatoria. A menudo se expresa $t$ como la periodicidad y $X(t)$ como el estado del proceso en el tiempo $t$ (Ross, 1996). En el caso de que el estado $X(t+1)$ dependa de $X(t)$, se le conoce como un proceso de Markov, y se usa en períodos sucesivos donde no hay certeza del estado en un momento cualquiera.

En el caso del precio de las opciones, estas se caracterizan por seguir un proceso estocástico llamado "Movimiento Geométrico Browniano (MGB)", el cual consiste, en mostrar la relación entre los precios actuales para el activo, y los posibles valores que pueden tomar en el futuro. El movimiento browniano es un objeto familiar para cada operador de opciones desde la aparición de B\&S (Tankov \& Voltchkova, 1992).

Siguiendo con el MDSM en lo que concierne a los "saltos", hace referencia al proceso Poisson,

el cual, se puede definir como una secuencia de variables exponenciales independientes $\left\{\theta_{i}\right\}_{i \geq 1}$ con un parámetro $\mu$ y $T_{n}=\sum_{i=1}^{n} \theta_{i}$, entonces el proceso 


$$
N_{t}=\sum_{n \geq 1} \mathbf{1}_{t \geq T_{n}}
$$

Se le considera como un proceso Poisson con parámetro $\mu$. En general las trayectorias del proceso Poisson son constantes a trozos y con saltos de uno en los tiempos $T_{i}$, y cada espacio entre los tiempos se distribuye de manera exponencial. Lo anterior, implica que, para cada $t>0$ la variable aleatoria $N_{t}$ se distribuya Poisson con parámetro $\mu_{t}$. Este proceso guarda con el MGB la particularidad de estacionariedad en los incrementos, es decir que, para cada tiempo mayor a otro, el incremento en la variable aleatoria $N_{t}$ en ambos tiempos es independiente de la historia del proceso hasta el tiempo mayor a $t$. Los procesos con intendencia y estacionariedad se les considera como procesos de Lévy.

En la aplicación financiera usualmente es raro encontrar un solo salto posible en procesos que se tienen; por lo tanto, se debe utilizar una generalización del proceso Poisson llamado "Proceso Poisson Compuesto (PPC)", en el cual, los intervalos entre los saltos periódicos se componen exponencialmente, y cada salto puede tener una distribución arbitraria.

Sea $A$ un proceso Poisson con un parámetro $\mu$, entonces:

$$
X_{t}=\sum_{i=1}^{N_{t}} Y_{i}
$$

donde $\left\{Y_{i}\right\}_{i \geq 1}$ es una secuencia de variables aleatorias; a $X_{t}$ se le conoce como PPC donde sus saltos son aleatorios y sus incrementos son independientes y estacionarios. La ley que gobierna el proceso en el tiempo $t$ no es explícita, pero su función característica tiene la siguiente forma:

$$
E\left(e^{i u X_{t}}\right)=\exp \left\{t \mu \int_{-\infty}^{\infty}\left(e^{i u x}-1\right) f(d x)\right\}
$$

donde la función característica tiene un rol importante en los procesos de difusión y saltos por su forma explícita del proceso.

En cuanto a los procesos de difusión con saltos y Lévy se puede afirmar lo siguiente: al combinar el MGB con una parte de los procesos de Poisson, se genera un proceso, donde los saltos tienen una evolución aleatoria entre los intervalos de tiempo como se muestra a continuación:

$$
X_{t}=\alpha t+\sigma B_{t}+\sum_{i=1}^{N_{t}} Y_{i}
$$

Y en donde, el modelo mejor conocido es el de Merton, en el cual, cada precio se puede expresar como $S_{t}=S_{0} e^{X_{t}}$ con saltos $Y_{t}$ normales. Al final la forma general para representar los procesos de levy $X_{t}=\alpha t+\sigma B_{t}+Z_{t}$, donde $Z_{t}$ es el proceso encargado de provocar los saltos.

$\mathrm{Al}$ ser los rendimientos de los precios de las opciones calculados como $r_{t}=\ln \left(\frac{P_{t}}{P_{t-1}}\right)$, los precios de los subyacentes son tratados con procesos de levy exponenciales para asegurar 
independencia y estacionaridad en los rendimientos. En este caso los saltos difusos corresponden a la siguiente expresión:

$$
S_{t}=S_{0} \cdot \exp \left\{\alpha t+\sigma B_{t}+\sum_{i=1}^{N_{t}} Y_{i}\right\}
$$

En donde los procesos tienden a ser un MGB después de cada salto, al ser valoración de opción se añade la tasa de interés en la definición del modelo de levy como $S_{t}=S_{0} \cdot \exp \left\{r_{t}+X_{t}\right\}$. Según Tankov \& Voltchkova (1992) este modelo puede ser considerado como una generalización de B\&S teniendo en cuenta la siguiente diferenciación:

$$
\frac{\partial S_{t}}{S_{t}^{(J)}}=\tilde{\alpha} d t+\sigma d B_{t}+d I_{t}
$$

Donde $I_{t}$ es un proceso Poisson compuesto y $S_{t}^{(J)}$ corresponde donde el salto fue realizado. Una serie de anotaciones realizadas por Tankov \& Voltchkova (1992) describen que los modelos conformados por los procesos de levy resultan insuficientes en algunos casos debido a los incrementos estacionarios de los rendimientos de los activos al tener un horizonte de precios estático y a su vez la dependencia de los estados de $X_{t}$ dado el horizonte de tiempo con momentos previamente definidos, los cuales en ocasiones no coinciden con la dependencia temporal de las observaciones. Una solución ilustrada es la combinación de modelos con saltos que guarden la volatilidad estocástica.

\subsection{Modelo de Difusión con saltos de Merton (MDSM)}

Una importante propiedad de B\&S, radica en no involucrar de manera directa en sus parámetros, el riesgo provocado por las preferencias de los inversionistas, por lo que se considera que este hace suposiciones neutrales frente al riesgo. En este modelo, el parámetro asociado a la volatilidad se asume como constante; sin embargo, diferentes análisis empíricos han demostrado que la volatilidad implícita de los activos tiende a variar con la fecha de maduración del activo y su precio strike (Gugole, 2016).

MDSM resulta como una extensión de la noción propuesta anteriormente por el de B\&S con la inclusión de saltos discretos en la definición de un movimiento browniano. En este proceso, el intervalo de llegada de los saltos sigue las propiedades del proceso compuesto de Poisson (intervalos exponenciales y saltos periódicos de manera normal).

\subsection{Modelo de Black y Scholes (B\&S)}

B\&S es el modelo matemático para la valoración de opciones creado en el siglo XIX, cuando el científico Robert Brown, después de ver que movimientos del polen en el agua describían un movimiento particular (movimiento browniano), esta clase de movimiento es usado en la 
matemática para describir y modelar comportamientos aleatorios; su descubrimiento permitió cimentar las bases del calculo estocástico donde se definen tasas de variación o cambio en donde uno o mas términos en las funciones tiene un componente aleatorio y puede ser descrito por medio de la probabilidad.

B\&S tomaron dos aproximaciones para hallar los precios de las opciones: la primera utiliza el modelo (Capital Asset Pricing Theory), y la otra utiliza el cálculo estocástico y la ecuación de difusión de calor con un cambio de variables. La solución propuesta a través del cálculo estocástico fue la realización de una ecuación diferencial que fue resuelta eventualmente a su proposición después de que encontraran que las propiedades del movimiento del calor, al pasar por un objeto, gozaban la misma particularidad que la valoración de los precios con su ecuación (Merton \& VenegasMartinez, 2021).

En general la valoración de opciones "call” con B\&S puede escribirse con cinco variables:

- $\quad S$ : Precio del subyacente en el mercado

- $\quad K$ : Precio de ejercicio (Strike)

- $\quad t$ : Tiempo (americana, en principio o en cualquier momento), (europea al final)

- $\quad C$ : Precio (Prima) de la opción

- $\quad r$ : Tasa libre de riesgo correspondiente al tiempo de vida de la opción

- $\sigma$ : La volatilidad o varianza correspondiente del logaritmo del precio de la acción, desviación estándar.

$$
C=f(S, K, t, \sigma, r)
$$

En general B\&S cuenta con las siguientes particularidades:

- El subyacente es una acción que no paga dividendos durante la vida del contrato

- El precio del subyacente se comporta como un MGB, es decir los precios siguen una distribución lognormal y los rendimientos una distribución normal.

- Las ventas en corto activo subyacente están permitidas

- No hay costos de transacción

- Existe un mercado de crédito donde se puede prestar o pedir prestado a una tasa libre de riesgo.

- La información es simétrica

La anterior descripción del modelo permite que sea posible escribirlo de la siguiente manera:

$$
C=S \cdot \Phi\left(d_{1}\right)-k e^{-r t} \cdot \Phi\left(d_{2}\right)
$$

Donde

$$
=\frac{\ln (S / K)+\left(r+\frac{\sigma^{2}}{2}\right) t}{\sigma \sqrt{t}}
$$




$$
d_{2}=\frac{\ln (S / K)+\left(r-\frac{\sigma^{2}}{2}\right) t}{\sigma \sqrt{t}}
$$

Donde $\Phi\left(d_{1}\right)$ y $\Phi\left(d_{2}\right)$, son distribuciones normales acumuladas.

En B\&S el precio evoluciona conforme a la siguiente ecuación estocástica (SDE) mencionada previamente:

$$
\left\{\begin{array}{c}
S_{t=0}=S_{0}>0 \\
d S_{t}=\mu S_{t} d t+\sigma S_{t} d W_{t}
\end{array}\right.
$$

Donde $W_{t}, t \in \mathbb{R}_{+}$, es llamado como un proceso Wiener o la versión estándar de un movimiento browniano (Gugole, 2016). El término $\mu S_{t} d t$ es conocido como parámetro de tendencia y conforma la parte determinística del proceso, en cuanto al término $\sigma S_{t} d W_{t}$ es propuesto para dar una descripción del precio de la acción y la volatilidad es usada para controlar el tamaño de las fluctuaciones del precio (estocasticidad).

Se puede resolver la ecuación diferencial estocástica (SDE) planteada por medio de la siguiente solución:

$$
S_{t}=S_{0} \cdot \exp \left(\sigma W_{t}+\left(\mu-\frac{\sigma}{2}\right) t\right)
$$

Donde $S_{t}$ es lognormal para $t>0$, y $S$ se puede describir como un proceso de markov. De esta manera tenemos que:

$$
\ln \left(S_{t}\right)=\ln \left(S_{0}\right)+\sigma W_{t}+\left(\mu-\frac{\sigma}{2}\right) t
$$

Esta expresión resulta mas cómoda para la estimación del precio de las opciones en cuanto a la computación, y permite identificar que el logaritmo de los rendimientos se comporta de manera normal.

$$
\ln \left(\frac{S_{t}}{S_{0}}\right) \sim N\left(\left(\mu-\frac{\sigma^{2}}{2}\right) t, \sigma^{2} t\right)
$$

(Gugole, 2016).

En cuanto al MDSM la similitud con la anterior ecuación diferencial estocástica se puede definir como:

$$
d S_{t}=(\mu-\lambda k) S_{t} d t+\sigma S_{t} d W_{t}+S_{t} d P_{t}
$$

Donde $P_{t}$, es un proceso Poisson compuesto con una tasa $\lambda$ (parámetro de intensidad), este proceso puede escribirse también como $P_{t}=\sum_{i=1}^{N_{t}}\left(Y_{i}-1\right), 0 \leq t \leq T . \quad P_{t}, Y_{i}, N_{t}$, son variables 
independientes. En la solución de la SDE definida sobre la dinámica del activo, $V_{i}=\ln \left(Y_{i}\right)$ se distribuye normal con media y varianza $m, \delta^{2}$ respectivamente y $k=\mathbb{E}\left[Y_{i}-1\right]=\exp \left\{m+\frac{\delta^{2}}{2}\right\}-1$. Se asume particularmente que las variables $V_{i}$ son independientes debido a que el precio asociado con cada salto sucede de manera aleatorio a un tiempo mayor a 0; cabe resaltar que otros investigadores parte de la idea de asumir a $V_{i}$ como doble exponencial diferente al supuesto de normalidad idealizado por Merton (Kou, 2008).

Conforme a la ecuación y descripción descrita anteriormente, la solución a esta SDE se presenta como:

$$
S_{t}=S_{0} \cdot \exp \left\{\sigma W_{t}+\left(\mu-\frac{\sigma^{2}}{2}-\lambda k\right) t\right\} \prod_{i=1}^{N_{t}} Y_{i}
$$

Teniendo en cuenta $V_{i}=\ln \left(Y_{i}\right) \sim N\left(m, \delta^{2}\right)$ se tiene que:

$$
X_{t}=\ln \left(\frac{S_{t}}{S_{0}}\right)=\left(\mu-\frac{\sigma^{2}}{2}-\lambda k\right) t+\sigma W_{t}+\sum_{i=1}^{N_{t}} V_{i}
$$

Donde los procesos $W, N$ y la variable aleatoria $V_{i}$ son multualmente independientes. Una manera posible para encontrar la distribución para $X_{t}$, se debe tener en cuenta la discretización planteada por (Khaldi, Djeddour, \& Meddahi, 2014) sobre el intervalo $(t, t+\Delta)$ es:

$$
\Delta X_{t}=\left(\mu-\frac{\sigma^{2}}{2}-\lambda k\right) \Delta+\sigma \Delta W_{t}+\sum_{i=1}^{\Delta N_{t}} V_{i}
$$

Donde la distribución $\Delta X_{t}$ se puede expresar como una ponderación entre densidades normales y la probabilidad de que $i$ saltos ocurran (condición de los rendimientos sobre el evento $\left\{N_{t}=i\right\}$, lo que con $\Delta=1$ conlleva a la siguiente expresión:

$$
X_{t}=\left(\frac{S_{t}}{S_{0}}\right) \sim N\left(\left(\mu-\frac{\sigma^{2}}{2}-\lambda k\right) t+i m, \sigma^{2} t+i \delta^{2}\right)
$$

La densidad de probabilidad para $\Delta X_{t}$ tiene la siguiente forma:

$$
\begin{aligned}
P\left(\Delta X_{t}\right)=f(x) & =\sum_{i=1}^{\infty} \frac{(\lambda \Delta)^{i}}{i !} e^{-\lambda \Delta} \\
& \cdot\left[\frac{1}{\sqrt{2 \pi\left(\sigma^{2} \Delta+i \delta^{2}\right)}} \exp \left(-\frac{\left.\left(x-\left(\mu-\frac{\sigma^{2}}{2}-\lambda k\right) \Delta-i m\right)^{2}\right)}{2\left(\sigma^{2} \Delta+i \delta^{2}\right)}\right)\right]
\end{aligned}
$$


Se puede notar que si $\theta=0, \Delta=1$, los saltos resultan ser simétricos y la distribución permite una mejor deducción para la estimación de los parámetros por el método de momentos como en el caso de (Khaldi, Djeddour, \& Meddahi, 2014).

En cuanto al logaritmo natural de los precios, se puede encontrar de la siguiente forma analítica:

$$
\ln \left(S_{t}\right)=\ln \left(S_{0}\right)+\left(\mu-\frac{\sigma^{2}}{2}-\lambda k\right) t+\sigma W_{t}+\sum_{i=1}^{N_{t}} V_{i}
$$

Donde la densidad de probabilidad del logaritmo de los precios es:

$$
P\left(\ln \left(S_{t}\right)\right)=\sum_{i=1}^{\infty} P\left(N_{t}=i\right) P\left(\ln \left(S_{t}\right) \mid N_{t}=i\right)
$$

Donde $P\left(N_{t}=i\right)$ es la probabilidad de salto en el precio del activo $i$ veces durante un intervalo t. $P\left(\ln \left(S_{t}\right) \mid N_{t}=i\right)$ la distribución condicionada de $\ln \left(S_{t}\right)$ dada la probabilidad de los saltos. En ese sentido, la densidad de $\ln \left(S_{t}\right)$ sería la siguiente:

$$
P\left(\ln \left(S_{t}\right)\right)=\sum_{i=1}^{\infty} \frac{(\lambda \mathrm{t})^{i}}{i !} e^{-\lambda \mathrm{t}} \cdot N\left(\ln \left(S_{0}\right)+\left(\mu-\frac{\sigma^{2}}{2}-\lambda k\right) t+i m, \sigma^{2} t+i \delta^{2}\right)
$$

La distribución citada es importante en cuanto a la disposición de los parámetros necesarios para modelar las series de tiempo -la estimación en el espacio de parámetros $\Theta=\{\lambda, \sigma, \delta, m\}$, los cuales, se puede lograr mediante máxima verosimilitud, estimación por momentos, estimación por PT (Passage Time) (Khaldi, Djeddour, \& Meddahi, 2014), y metodologías bayesianas (Tunaru \& Zheng, 2017)-.

Los supuestos descritos anteriormente, permiten la flexibilidad suficiente para realizar estimaciones bayesianas sobre los parámetros del modelo, ya que como bien se mencionó el logaritmo de los saltos se asume como normal.

Existen otras formulaciones sobre MDSM, en algunos casos se toma el componente del parámetro de tendencia (determinístico) de la SDE sobre la dinámica $d S_{t}$ con un único parámetro $\mu=r-\lambda k$, resultando en la siguiente expresión:

$$
\begin{gathered}
\frac{d S_{t}}{S_{t}}=\mu d t+\sigma W_{t}+d P_{t} \\
d S_{t}=\mu S_{t} d t+\sigma S_{t} W_{t}+S_{t} d P_{t}
\end{gathered}
$$

Donde $\mu$ y $\sigma$ se asumen como constantes para la parte determinística y estocástica del proceso. La solución de la SDE en este caso se considera como: 


$$
S_{t}=S_{0} \cdot \exp \left\{\sigma W_{t}+\left(\mu-\frac{\sigma^{2}}{2}\right) t\right\} \prod_{i=1}^{N_{t}} Y_{i}
$$

Al tomar una discretización usando unidades la ecuación $\frac{d s_{t}}{s_{t}}$, puede ser aproximada como:

$$
\frac{\Delta S_{t}}{S_{t}}=\mu+\sigma Z+B \cdot X
$$

Donde $B, X, Z$ son variables aleatorias independientes, $B$ siendo una distribución Bernoulli y $\mathrm{Z}$ normal. Esta forma de discretización fue propuesta como medida de detección a los saltos del proceso en un contexto bayesiano por (Rifo \& Torres, 2009).

En el caso $X$ se sigue una distribución bernoulli con parámetro desconocido $p$ y $B X$ una distribución de la siguiente manera:

$$
f(x)=\left\{\begin{array}{l}
\lambda p, \quad \text { para } x=1 \\
1-\lambda p, \text { para } \mathrm{x}=0
\end{array}\right.
$$

En el caso de la distribución de densidad para $\mu+\sigma Z+B X$, se tiene:

$$
f(y)=\frac{1}{\sqrt{2 \pi \sigma^{2}}}\left((1-\lambda p) \exp \left(-\frac{1}{2 \sigma^{2}}(y-\mu)^{2}+\lambda p \cdot \exp \left(-\frac{1}{2 \sigma^{2}}(y-(\mu+1))^{2}\right)\right)\right)
$$

(Rifo \& Torres, 2009)

\section{Estudio de simulación para la estimación}

Conforme al objetivo propuesto en este trabajó, que se focaliza en encontrar forma de implementar la estimación bayesiana para los parámetros para el comportamiento asociado a los procesos estocásticos de MDSM y B\&S.

En este orden de ideas, durante los siguientes procedimientos se buscará explicitar una comparación entre distintos modelos para el ajuste y modelamiento del logaritmo de los rendimientos, con el objetivo de realizar un análisis sobre las estimaciones e intervalos de credibilidad de los parámetros, y posteriormente resaltar la importancia de la estimación bayesiana sobre MDSM tradicional y B\&S. En ese sentido, se buscará evidenciar de una manera óptima la estimación de los parámetros diferente a la selección arbitraria de estos mismos en la simulación de los procesos asociados a ambos modelos.

Para realizar las simulaciones de los procesos anteriormente mencionados se tomaron las acciones de Apple, Nvidia y Tesla los últimos 252 días de precios de negociación (hasta el 23 de junio de 2021) con el objetivo de ilustrar como la inclusión de los componentes aleatorios en forma de saltos, resulta ser más apropiada para el modelamiento del precio de los rendimientos, y a su vez mostrar por qué vía la estadística bayesiana sobre los parámetros, puede ser recomendada en las 
estimaciones de los parámetros asociados a los procesos estocásticos de los modelos anteriormente mencionados.

A continuación, se muestra el comportamiento histórico del precio de la acción de Apple, Nvidia y Tesla en el período del 23 de mayo de 2014 al 23 de junio de 2021.

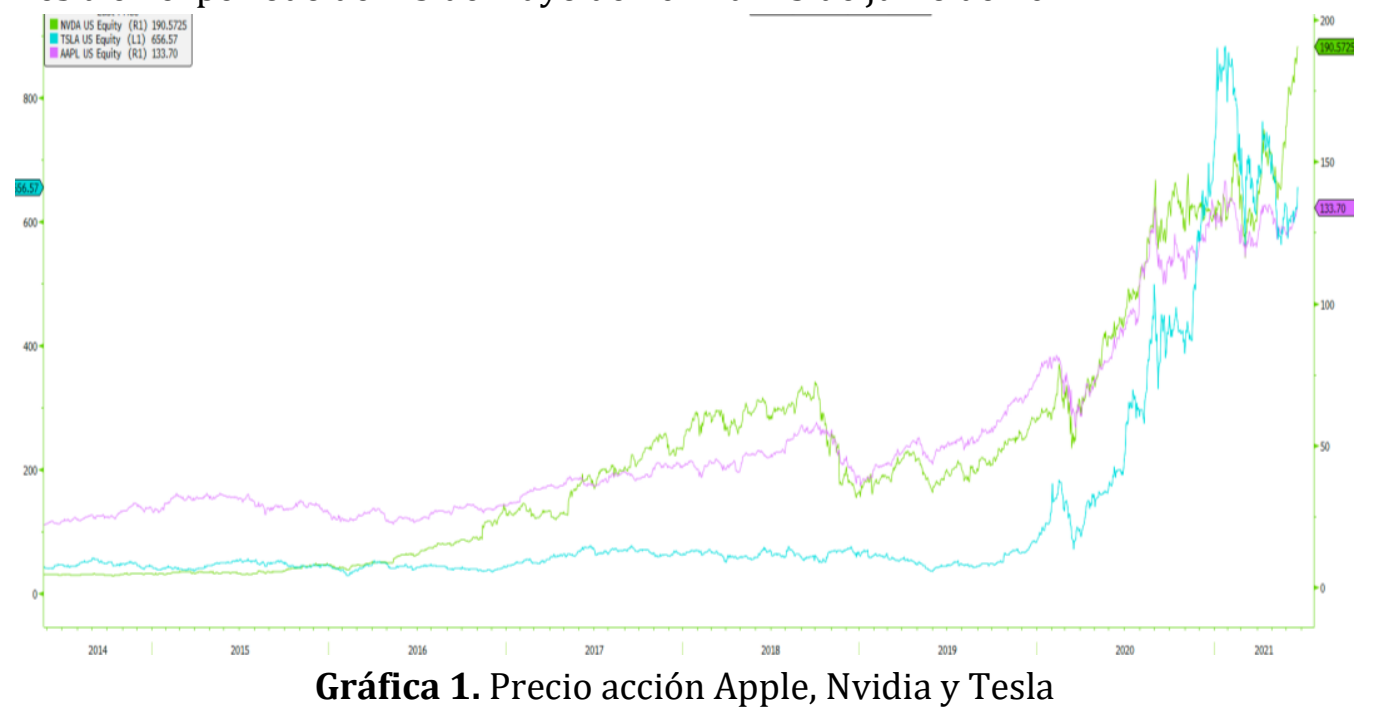

Fuente: Pantallazo tomado de la Plataforma Bloomberg el 23 de junio de 2021

Se puede evidenciar en el pantallazo de la plataforma Bloomberg que la acción de Apple ha presentado un comportamiento mixto durante el tiempo descrito no solamente por la situación generada por el COVID-19, sino también de alguna manera por la escasez de silicio que ha afectado las ventas de iPhone.

Cabe de igual manera resaltar, que los precios de las acciones tecnológicas sufrieron al igual que el mercado de renta variable, los efectos de la pandemia producida por el COVID-19; sin embargo, a pesar de esta coyuntura internacional, se ha evidenciado en el mercado mayor rentabilidad en las acciones de Tesla y Nvidia, como se puede observar en la siguiente gráfica:

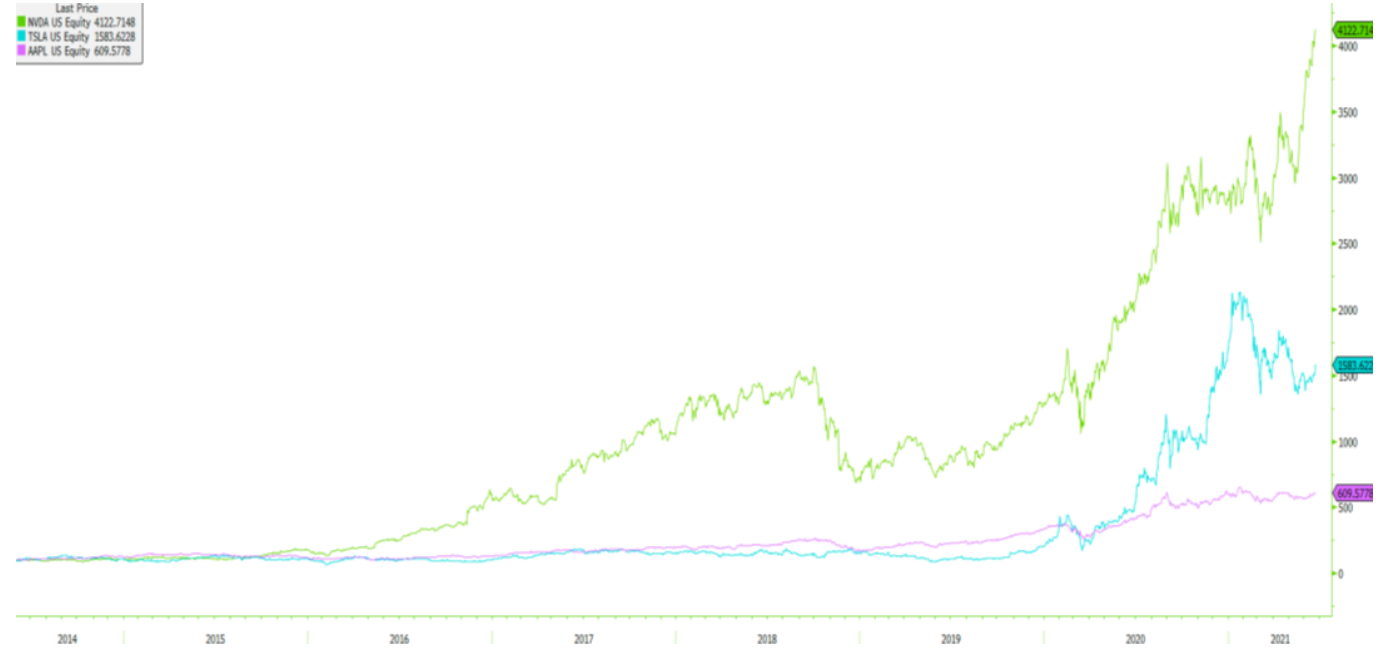

Gráfica 2. Rentabilidad acción Apple, Nvidia y Tesla (período 23/mayo/2014 al 23/junio/2021) Fuente: Pantallazo tomado de la Plataforma Bloomberg el 23 de junio de 2021 
En el marco temporal descrito, se puede observar que la mayor rentabilidad en el período del 23 de mayo de 2014 al 23 de junio de 2021 la obtuvo la acción de Nvidia con un 4122\% como consecuencia de la decisión de lanzamiento de una nueva línea de tarjetas gráficas que cuentan con la característica de potencializar los juegos electrónicos y sus transmisiones en línea.

Finalmente, los movimientos en las empresas de tecnología ha sido de naturaleza hacia al alza con excepción de marzo de 2020, que fue el pronunciamiento a nivel mundial de la Organización Mundial de la Salud, la cual manifestó que el mundo se encontraba amenazada por el COVID-19, el cual generó el desplome la mayoría de todos los activos del mercado de renta variable a nivel global y un apetito refugio en los bonos del tesoro americano correspondiente a los bonos de 10 años; no obstante, el auge de las tecnologías vía inteligencia artificial así como el desarrollo del comercio electrónico, ha propiciado el incrementó del precio de las acciones de empresas vinculadas al sector tecnológico.

De otro lado, se puede evidenciar que con un p-valor arrojado, y un nivel de confianza del $95 \%$, se demuestra que los rendimientos de cada una de las acciones citadas contaron con la característica de ser estacionarios $(P-$ valor $\leq 0,01)$

Con base en lo anterior, se calcularon los correlogramas de autocorrelación y autocorrelación parcial con un máximo de 30 rezagos en el tiempo para cada una de las acciones.

De conformidad con los correlogramas de cada una de las acciones se escogió el siguiente modelo:

Cuadro 1. Modelos ARIMA una aproximación inicial

\begin{tabular}{|c|l|l|l|}
\hline Acción & \multicolumn{1}{|c|}{ Modelo } & \multicolumn{1}{|c|}{ Estadístico } & \multicolumn{1}{|c|}{ P-valor(z) } \\
\hline Apple & Arima $(0,1,1)$ & -0.999981 & $<2.2 \mathrm{e}-16$ \\
\hline Nvidia & Arima $(0,1,1)$ & -1.000000 & $<2.2 \mathrm{e}-16$ \\
\hline Tesla & Arima $(0,1,1)$ & -0.987554 & $<2.2 \mathrm{e}-16$ \\
\hline
\end{tabular}

Fuente: Elaboración propia

De acuerdo con, el p-valor arrojado para cada una de las acciones, con un nivel de confianza del 95\%, se puede afirmar que el modelo Arima $(0,1,1)$ es significativo.

En lo que corresponde a la prueba de normalidad a continuación se muestra la prueba de normalidad Jar-Quebera, que tiene como objetivo conocer, si los residuales derivados del mejor modelo en cada una de las acciones (Apple, Nvidia y Tesla) se distribuyen con respecto a la distribución de probabilidad normal para los residuales.

Cuadro 2. Prueba de normalidad para los residuales

\begin{tabular}{|c|c|c|c|}
\hline Acción & Estadístico de prueba & Grados de libertad & P-valor \\
\hline Apple & 70.246 & 2 & $5.551 \mathrm{e}-16$ \\
\hline Nvidia & 20.092 & 2 & $4.336 \mathrm{e}-05$ \\
\hline Tesla & 172.72 & 2 & $<2.2 \mathrm{e}-16$ \\
\hline
\end{tabular}

Fuente: Elaboración propia 
De acuerdo con el p-valor arrojado para cada uno de los instrumentos financieros, con un nivel de confianza del 95\%, se puede afirmar que los residuales del mejor modelo no se distribuyen con respecto a la distribución de probabilidad normal.

En lo que atañe a la prueba de heterocedasticidad de McLeod-Li que tiene como objetivo conocer si las varianzas de los residuales del mejor modelo en cada una de las acciones, si son iguales o diferentes a lo largo del tiempo (Esta prueba se realizó teniendo en cuenta un rezago en el tiempo) arrojó los siguientes resultados:

Cuadro 3. Prueba de heterocedasticidad de los residuales

\begin{tabular}{|c|c|}
\hline Acción & P-valor \\
\hline Apple & 0.7997394 \\
\hline Nvidia & 0.0270482529 \\
\hline Tesla & 0.15412589 \\
\hline
\end{tabular}

Fuente: Elaboración propia

De conformidad con el cuadro 3, el p-valor arrojado con un nivel de confianza del 95\%, se puede afirmar que, los residuales del mejor modelo no poseen diferentes varianzas a lo largo del tiempo. Lo anterior quiere decir, que las varianzas de los residuales son iguales; por lo tanto, los rendimientos de la acción de Apple y Tesla no son volátiles.

En lo que concierne a la acción de Nvidia con el p-valor arrojado, con un nivel del 95\%, se puede afirmar que los residuales del mejor modelo poseen diferentes varianzas a lo largo del tiempo. Lo explicitado quiere decir, que las varianzas de los residuales no son iguales; por lo tanto, los rendimientos de cada una de las acciones son volátiles.

Para analizar el MDSM es necesario realizar distintas simulaciones con diversas combinaciones de parámetros, con el objetivo de encontrar y explicar de que manera óptima puede comportarse el mercado en el futuro; para esto, utilizando métodos implementados por (Khaldi, Djeddour, \& Meddahi, 2014), se realizó una simulación inicial sobre la asunción de los supuestos en B\&S y MDSM; para tal efecto, se comparan los procesos simulados del B\&S en la gráfica 3 y los caminos simulados por el modelo MDSM en la gráfica 4 para el histórico de precios de la acción Nvidia hasta el 2018. Este período en específico se tomó en el caso de la acción Nvidia, como consecuencia del auge de nuevas tecnologías relacionadas al hito de la inteligencia artificial.

De acuerdo con lo anterior, se procedió a recortar la serie con el objetivo de presentar los saltos de los procesos simulados en este período por el modelo MDSM, ya que, desde 2016 (ultimo 25\% del total de los precios, 2016-2018) se presentó el efecto de "salto" o "alza", el cual, el modelo MDSM explica mejor el comportamiento de los precios; por lo tanto, se graficó el espejo de la serie original por objetivos de visualización. 


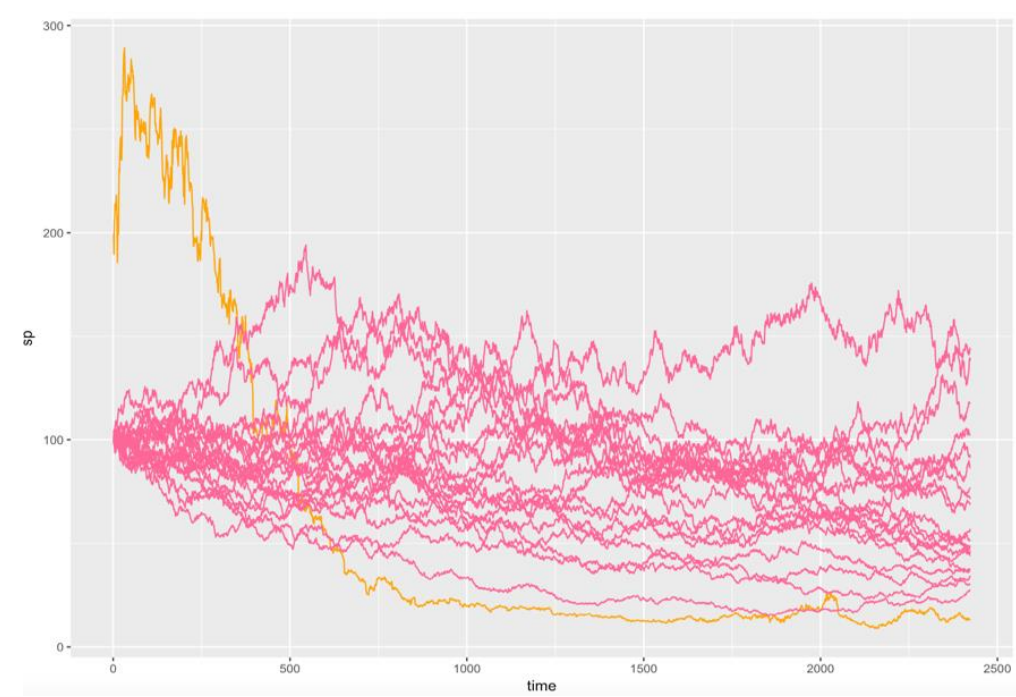

Gráfica 3. Simulación proceso B\&S en la acción de Nvidia

Fuente: Elaboración propia

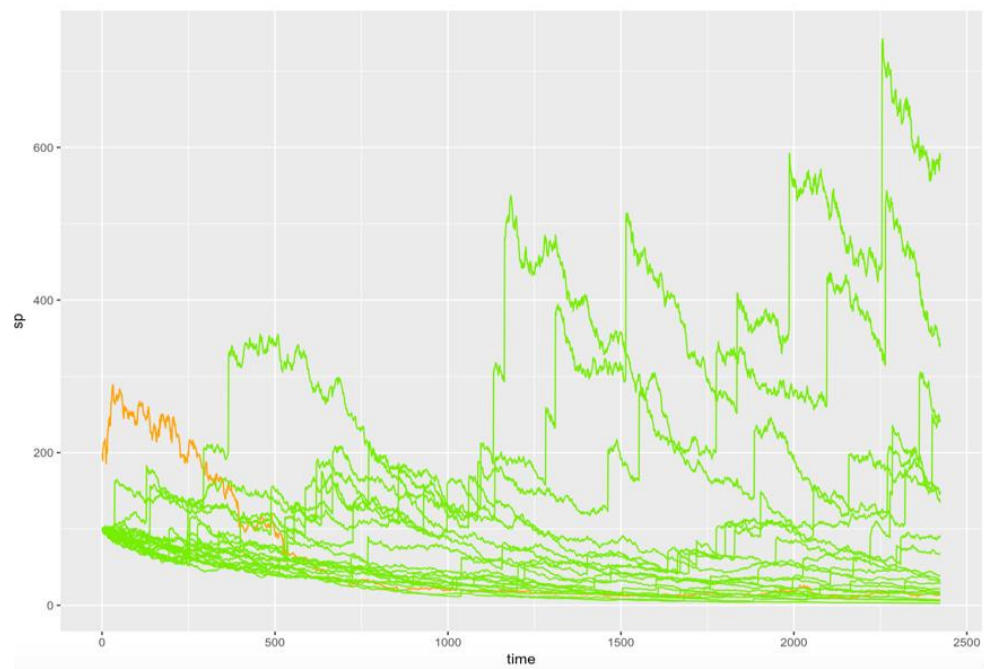

Gráfica 4. Simulación modelo de difusión con saltos en la acción de Nvidia

Fuente: Elaboración propia

Como se puede observar en la gráfica 3 y 4, en el caso de B\&S al no contar con una diferenciación entre los saltos a manera de un proceso Poisson, no detecta de manera amplia los cambios en el precio de la acción, debido a que, la composición que presenta arroja un MGB ; En el caso de la inclusión de saltos, los cambios son más marcados y volátiles.

Los parámetros relacionados al parámetro de tendencia, y a la parte estocástica de los procesos simulados suelen ser determinados de manera empírica, como se puede observar en las gráficas 3 y 4.

Cabe resaltar que, el objetivo del presente trabajo es proporcionar soluciones a la estimación de los parámetros para B\&S y MDSM de manera bayesiana generando intervalos de credibilidad asociados a los parámetros, con el fin de generar simulaciones del proceso, más cercanas al 
comportamiento del logaritmo natural de los rendimientos para cada una de las acciones: Apple, Nvidia y Tesla.

\subsection{Estimaciones sobre los parámetros}

De acuerdo con el desarrollo investigativo, se busca por medio de la estimación bayesiana encontrar una propuesta mejor a la estadística clásica usada en la estimación de parámetros para MDSM en yuxtaposición con B\&S; para tal efecto se sigue la estimación con la siguiente propuesta en parámetros y distribución:

Para la estimación de los parámetros se utiliza el muestreador de Gibbs; este es un algoritmo (MCMC) Markov Chain Monte Carlo usado para la obtención de la distribución de probabilidad de los parámetros usados, cuando se requiere realizar muestras de la distribución posterior calculada, a partir de la verosimilitud y las distintas distribuciones a priori asociadas a cada parámetro a estimar.

Dada la distribución posterior:

$$
P\left(\Theta \mid x_{1}, \ldots, x_{t}\right)=\frac{P\left(x_{1}, \ldots, x_{t} \mid \Theta\right) P(\Theta)}{\int_{\Theta} P\left(x_{1}, \ldots, x_{t} \mid \Theta\right) P(\Theta)}
$$

Donde $\Theta$ es el espacio de parámetros, $P\left(x_{1}, \ldots, x_{t} \mid \Theta\right)$ se le conoce como la verosimilitud de los datos, $P(\Theta)$ son las distribuciones asociadas al espacio de parámetros y $\int_{\Theta} P\left(x_{1}, \ldots, x_{t} \mid \Theta\right) P(\Theta)$ se ele conoce como constante de normalización.

El muestreador de Gibbs funciona como un algoritmo usando recursión entre muestras generadas por las distintas distribuciones condicionales asociadas al espacio de parámetros $\Theta$.

\section{A. Modelo de Black y Scholes (B\&S)}

Para la estimación de los parámetros de B\&S es necesario recalcar las características y supuestos previamente mencionados sobre los movimientos geométricos brownianos.

Al ser $x$ un MGB descrito por la ecuación estocástica:

$$
d S_{t}=\mu S_{t} d_{t}+\sigma S_{t} B_{t}
$$

Con solución descrita como:

$$
S_{t+1}=S_{t} \exp \left(\mu-\frac{1}{2} \sigma^{2}+\sigma\left(B_{t+1}-B_{t}\right)\right)
$$

Donde los rendimientos son distribuidos de manera normal:

$$
R_{t}=\ln \left(\frac{S_{t * 1}}{s_{t}}\right) \sim N\left(\mu-\frac{1}{2} \sigma^{2}, \sigma^{2}\right)
$$


Siguiendo la reparametrización $\beta=\mu-\frac{1}{2}$, las distribuciones a priori de los parámetros pueden ser halladas fácilmente (Jones, Simon, \& Hall Simon, 1998). El espacio de parámetros definido en este caso consiste en el siguiente conjunto $\Theta=\{\beta, h\}$. En el caso de esta investigación se proponen las siguientes priors:

$$
\begin{gathered}
\beta \sim N\left(0, e^{-6}\right) \\
h \sim \operatorname{Gamma}_{I}(0.1,0.5)
\end{gathered}
$$

donde $h=\frac{1}{\sigma^{2}}$, es la precisión. Por medio del muestreador de Gibbs con Rjags (Plummer, JAGS Version 4.3.0 User manual, 2017), (Plummer, CRAN R, 2019). usando 10000 iteraciones, 10 cadenas, removiendo las primeras 1000 observaciones para evitar el efecto del valor inicial (burning), tomando cada 5 observaciones (thinning), se obtuvieron los siguientes resultados sobre las estimaciones bayesianas e intervalos de más alta densidad para los parámetros del componente determinístico y estocástico del proceso para cada una de las acciones:

Cuadro 4. Estimación bayesiana parámetros B\&S, logaritmo natural de los rendimientos de Apple, Nvidia y Tesla

\begin{tabular}{|c|c|c|c|c|c|}
\hline Acción & Parámetro & Estimación & Desviación & $\mathbf{2 . 5 \%}$ & $\mathbf{9 7 . 5 \%}$ \\
\hline \multirow{4}{*}{ Apple } & $\beta$ & 0.000 & 0.000 & $-2 \mathrm{e}-5$ & $3 \mathrm{e}-6$ \\
\cline { 2 - 6 } & $\sigma^{2}$ & 22.534 & 2.010 & 18.759 & 26.641 \\
\cline { 2 - 6 } & $\begin{array}{c}\text { desvío } \\
\text { (deviance) }\end{array}$ & -1231.215 & 2.057 & -1233.199 & -1225.810 \\
\hline \multirow{4}{*}{ Nvidia } & $\beta$ & 0.000 & 0.000 & $-2 \mathrm{e}-5$ & $3 \mathrm{e}-6$ \\
\cline { 2 - 6 } & $\sigma^{2}$ & 11.524 & 1.021 & 10.830 & 13.599 \\
\cline { 2 - 6 } & $\begin{array}{c}\text { desvío } \\
\text { Tesla }\end{array}$ & -1060.297 & 2.000 & -1060.907 & -1054.874 \\
\cline { 2 - 6 } & $\beta$ & 0.000 & 0.000 & $-2 \mathrm{e}-5$ & $3 \mathrm{e}-6$ \\
\cline { 2 - 6 } & $\begin{array}{c}\text { deviance) } \\
\text { (deviance) }\end{array}$ & -853.896 & 2.068 & -855.337 & -848.221 \\
\hline
\end{tabular}

Fuente: Elaboración propia

Dadas las configuraciones de distribuciones a priori, Se puede observar a continuación la estimación por perdida cuadrática de los parámetros B\&S de la siguiente manera: 


Cuadro 5. Estimación por perdida cuadrática del parámetro $\boldsymbol{\sigma}^{2}$ B
\begin{tabular}{|c|c|}
\hline Acción & Estimación de $\boldsymbol{\sigma}^{2}$ \\
\hline Apple & $\hat{\mu}=\hat{\beta}+\frac{1}{2} \hat{\sigma}^{2}=11.267$ \\
& $\hat{\sigma}^{2}=22.534$ \\
\hline Nvidia & $\hat{\mu}=\hat{\beta}+\frac{1}{2} \hat{\sigma}^{2}=11.524$ \\
& $\hat{\sigma}^{2}=5.762$ \\
\hline Tesla & $\hat{\mu}=\hat{\beta}+\frac{1}{2} \hat{\sigma}^{2}=5.128$ \\
& $\hat{\sigma}^{2}=2.564$ \\
\hline
\end{tabular}

Fuente: Elaboración propia

El cuadro 6 muestra los intervalos de más alta densidad, donde la probabilidad de que el verdadero parámetro $\beta$ se encuentre entre $-2 \mathrm{e}-5$ y $-3 \mathrm{e}-6$ es del $95 \%$ al igual que la probabilidad de que el verdadero valor de $\sigma^{2}$ para cada acción se encuentra en un rango que se detalla a continuación:

Cuadro 6. Intervalo de confianza del parámetro $\boldsymbol{\sigma}^{2} \mathrm{~B} \& \mathrm{~S}$

\begin{tabular}{|c|c|}
\hline Acción & Rango \\
\hline Apple & 18.759 a 26.641 \\
\hline Nvidia & 10.830 a 13.599 \\
\hline Tesla & 4.815 a 6.958 \\
\hline
\end{tabular}

Fuente: Elaboración propia

Para revalidar la efectividad de las estimaciones realizadas bajo el enfoque bayesiano usando el muestreador de Gibbs, las gráficas de los cuadros 7, 8 y 9 (ver apéndice) muestran el diagnóstico sobre las cadenas de Markov simuladas para la estimación de los parámetros de la distribución posterior asociada al modelo B\&S; estas gráficas permiten ver la efectividad de la estimación en términos de convergencia en distribución, autocorrelación y aleatoriedad de las distintas cadenas generadas por el algoritmo MCMC usado en Rjags.

En general, en las cadenas de Markov simuladas para los parámetros $\beta, \sigma^{2}$ (ver cuadros 7, 8 y 9 del apéndice), se pueden observar las distribuciones a posteriori asociadas a cada parámetro estimado, al igual que la aleatoriedad en las cadenas, en cuanto a la autocorrelación, para cada acción, en la cual los valores de las cadenas no presentan ningún tipo de patrón determinístico y el 75\% de los lags en el diagrama de autocorrelación se encuentran por debajo de la banda. El gráfico de convergencia muestra la media móvil usada para la estimación del valor final de cada parámetro.

La estimación del desvío (deviance) (ver cuadro 9), su convergencia e intervalos de más alta densidad para media a posteriori, indican la efectividad y el ajuste del modelo usado basado en la selección de las distribuciones a priori mencionadas anteriormente.

Una vez realizadas las estimaciones sobre los parámetros de $B \& S$, se realizan diferentes simulaciones del proceso sobre la serie del logaritmo natural de los rendimientos de cada una de las 
acciones, el cuadro 10, muestra en detalle un total de 5 caminos simulados sobre el logaritmo natural de los rendimientos (serie de color negro) usando los parámetros encontrados de manera bayesiana.

Una vez calculadas las estimaciones de los parámetros, se realizan diferentes caminos simulados sobre la serie original del logaritmo natural de los rendimientos para cada una de las acciones citadas (Apple, Nvidia y Tesla) como se puede evidenciar en el cuadro 10.

Los caminos simulados usando la distribución a posteriori aproximada con los parámetros encontrados no reflejan el comportamiento de la serie original (logaritmo natural de los rendimientos para cada acción) ya que se puede observar en las distintas graficas por cada acción, como las series asociadas a los procesos que simulan el comportamiento de B\&S presentan mayores fluctuaciones y no se parecen a la serie original (Ver cuadro 10).

\section{B. Modelo de Difusión con saltos de Merton (MDSM)}

Sea $\Theta=\{\lambda, \sigma, \delta, m, \mu\}$ el espacio de parámetros para la distribución de MDSM considerando

los rendimientos expresados como $R_{t}=\ln \left(\frac{S_{t * 1}}{S_{t}}\right)$. De esta manera se tiene la siguiente definición para la densidad posterior:

$$
\begin{gathered}
f\left(\Theta \mid x_{1}, \ldots, x_{t}\right) \propto f(\Theta) f\left(x_{1}, \ldots, x_{t} \mid \Theta\right) \\
f\left(\lambda, \sigma, \delta, r, m \mid x_{1}, \ldots, x_{t}\right) \propto f(\lambda) f(\sigma) f(\delta) f(\mu) f(m) f\left(x_{1}, \ldots, x_{t} \mid \lambda, \sigma, \delta, r, m\right) \\
f\left(x_{1}, \ldots, x_{t} \mid \lambda, \sigma, \delta, \mu, m\right)=f_{R_{\Delta t}}(x)=\sum_{k=0}^{\infty} p_{k}(\lambda \Delta t) N\left(x \mid\left(\mu-\frac{1}{2} \sigma^{2}\right) \Delta t+m k, \delta^{2} \Delta t+\sigma^{2} k\right)
\end{gathered}
$$

Donde $f(\lambda), f(\sigma), f(\delta), f(\mu)$ y $f(m)$ corresponden a las distribuciones prior de los parámetros a estimar y $f\left(x_{1}, \ldots, x_{t} \mid \lambda, \sigma, \delta, \mu, m\right)$ es la verosimilitud. En el caso $p_{k}(\lambda \Delta t)=$ $p_{k}\left(\Delta N_{t}=k\right)=\frac{(\lambda \Delta t)^{k}}{k !} e^{-\lambda \Delta t}$ (Tang, 2018). Es necesario precisar el supuesto de independencia sobre la información a priori sobre los parámetros del modelo. En este orden, con el objetivo de encontrar convergencia y ningún tipo de autocorrelación en las cadenas de las estimaciones bayesianas para los parámetros se utilizaron las siguientes propuestas prior:

$$
\begin{gathered}
\lambda \sim \operatorname{Beta}(2,5) \\
\mu \sim N(0,1) \\
m \sim N\left(0, e^{-04}\right)
\end{gathered}
$$

En el caso de los parámetros $\sigma$ y $\delta$, se uso la precision como medida de varianza para ambos parámetros, siendo $\sigma^{2}=\frac{1}{h} \mathrm{y} \delta^{2}=\frac{1}{J}$, donde:

$$
\begin{aligned}
& h \sim \operatorname{Gamma}_{I}(20,0.5) \\
& J \sim \operatorname{Gamma}_{I}(10,0.5)
\end{aligned}
$$


Teniendo en cuenta la descripción de los parámetros mencionados anteriormente, así como sus distribuciones a priori se usó nuevamente el muestreador de Gibbs con Rjags para realizar la estimación bayesiana usando 3 cadenas, 10000 iteraciones, quitando de la cadena al menos 1000 observaciones cada 5 iteraciones. Se muestra la siguiente tabla la estimación e intervalos de más alta densidad para los parámetros mencionados:

Cuadro 11. Estimación bayesiana parámetros del modelo MDSM, logaritmo natural de los rendimientos.

\begin{tabular}{|c|c|c|c|c|c|}
\hline Acción & Parámetros & Estimación & desviación & $2.5 \%$ & $97.5 \%$ \\
\hline \multirow{6}{*}{ Apple } & $\delta^{2}$ & 20.557 & 1.919 & 16.915 & 24.597 \\
\hline & $\lambda$ & 0.287 & 0.159 & 0.042 & 0.637 \\
\hline & $m$ & $-3.11 \mathrm{e}-05$ & $3.088 \mathrm{e}-05$ & $-4.034 \mathrm{e}-05$ & $5.573 e-05$ \\
\hline & $\mu$ & 0.014 & 0.005 & 0.005 & 0.024 \\
\hline & $\sigma^{2}$ & 0.026 & 0.006 & 0.017 & 0.040 \\
\hline & $\begin{array}{c}\text { Desvío } \\
\text { (deviance) }\end{array}$ & -1227.926 & 3.366 & -1231.788 & -1219.285 \\
\hline \multirow{6}{*}{ Nvidia } & $\delta^{2}$ & 10.531 & 0.976 & 8.711 & 12.519 \\
\hline & $\lambda$ & 0.287 & 0.161 & 0.041 & 0.646 \\
\hline & $m$ & 0.000 & 0.000 & 0.000 & 0.000 \\
\hline & $\mu$ & 0.011 & 0.006 & -0.001 & 0.023 \\
\hline & $\sigma^{2}$ & 0.026 & 0.006 & 0.017 & 0.041 \\
\hline & $\begin{array}{c}\text { Desvío } \\
\text { (deviance) }\end{array}$ & -1057.858 & 3.267 & -1061.652 & -1049.676 \\
\hline \multirow{6}{*}{ Tesla } & $\delta^{2}$ & 4.715 & 0.433 & 3.892 & 5.597 \\
\hline & $\lambda$ & 0.284 & 0.160 & 0.041 & 0.644 \\
\hline & $m$ & 0.000 & 0.000 & 0.000 & 0.000 \\
\hline & $\mu$ & 0.023 & 0.008 & 0.017 & 0.040 \\
\hline & $\sigma^{2}$ & 0.026 & 0.006 & 0.022 & 0.041 \\
\hline & $\begin{array}{c}\text { Desvío } \\
\text { (deviance) }\end{array}$ & -853.833 & $3-286$ & -856.223 & -845.374 \\
\hline
\end{tabular}

Fuente: Elaboración propia

En el caso de las anteriores estimaciones, se encontró un DIC (Criterio de información de desvío o Deviance information criterion) de -1409.6 a -848.4 (ver cuadro 12). Este valor indica la calidad del ajuste de la distribución a posteriori estimada, al logaritmo natural de los rendimientos; entre más bajo es el valor mayor es la calidad del ajuste. 
Cuadro 12. DIC Criterio de información de desvío o Deviance information criterion

\begin{tabular}{|c|c|}
\hline Acción & $\begin{array}{c}\text { DIC Criterio de información de desvío o } \\
\text { Deviance information criterio }\end{array}$ \\
\hline Apple & -1222.3 \\
\hline Nvidia & -1052.5 \\
\hline Tesla & -848.4 \\
\hline
\end{tabular}

Fuente: Elaboración propia

De otro lado, el DIC indica que la estimación planteada para cada una de las acciones fue la mejor, conforme a las diferentes propuestas para las distribuciones a priori de los parámetros mencionados anteriormente. Se puede observar en ese sentido que: en la estimación bayesiana, la desviación en la mayoría de los parámetros es cercana a 0 , lo que indica que, los intervalos de más alta densidad son más estrechos siendo un buen indicio de una estimación adecuada.

Para analizar la calidad de las estimaciones de los parámetros calculados en detalle, se realizaron las gráficas de convergencia para las estimaciones (ver cuadro 13, 14, 15, 16, 17 y 18 del apéndice) en donde se puede observar que tanto en las cadenas como la distribución se encuentra total convergencia, así como ningún problema de autocorrelación.

De conformidad con lo anterior, en la estimación de los parámetros, se buscó reafirmar la importancia de la estimación bayesiana frente a una selección arbitraria para cada MGB asociado al de B\&S, y los parámetros adicionales de salto asociados a los procesos de MDSM. En este caso, la estimación bayesiana logró delimitar de manera más detallada la selección el ajuste de los parámetros a los datos, ya que, amplia el espectro de posibilidades frente a la selección de un espacio aleatorio para los posibles valores de los parámetros.

Con el objetivo de validar de otro lado la eficacia de las estimaciones encontradas usando el muestreador de Gibbs para el cálculo de los parámetros del modelo MDSM, se simularon al igual que, en los cálculos de B\&S, distintos caminos aleatorios usando los parámetros encontrados, el cuadro 19 muestra los distintos caminos simulados con el objetivo de ilustrar el ajuste apropiado del modelo MDSM a la dinámica estocástica del logaritmo natural de los rendimientos.

Como se puede observar en el cuadro 19, los caminos simulados presentan comportamientos similares a la serie del logaritmo natural de los rendimientos tanto en su rango de valores y la variabilidad de los procesos. Comparando los cuadros 10 y 19 se puede evidenciar como el modelo MDSM se ajusta mejor a los datos a lo largo del tiempo, al igual que la volatilidad de la serie analizada en comparación al MGB caracterizado por del modelo B\&S.

En el cuadro 19 se puede observar en las distintas graficas por cada acción, como los caminos simulados usando la distribución asociada al modelo MDSM después de la estimación bayesiana realizada, capturan de manera efectiva la dinámica estocástica de la serie original (logaritmo natural de los rendimientos); esto se puede verificar, comparando visualmente la similitud de los caminos simulados con el comportamiento de la serie original para cada una de las acciones analizadas.

Se puede observar en el cuadro 19 que, para cada acción relacionada, como MDSM se aproxima a la realidad de los rendimientos en comparación al de B\&S.

De otro lado, con una aproximación mejor a la estimación de los parámetros por medio de métodos bayesianos, MDSM posee un mayor grado de ajuste sobre el comportamiento en distribución del logaritmo natural de los rendimientos. En ese sentido se puede observar 
explícitamente, como el modelo a través de diferentes procesos o caminos simulados se sobrepone a la distribución del logaritmo natural de los rendimientos reproduciendo su conducta leptocúrtica (ver cuadro 20).

Adicionalmente al método de estimación, se pudo observar, que el modelo al usar saltos aleatorios se aproxima de manera más exacta a la dinámica de los rendimientos en comparación a B\&S. En esa misma línea, se puede decir, que una de las razones por las que en modelos con saltos difusos reflejan de mejor manera eventos sobre series que son difíciles de predecir, es su capacidad de capturar el comportamiento volátil del precio de cualquier instrumento financiero en el mercado; por lo tanto, este tipo de característica no se puede tomar en cuenta con modelos basados en movimientos geométricos Brownianos como lo es el caso de B\&S.

\section{Conclusión}

Se logró con el desarrollo de esta investigación proponer distintas medidas en cuanto a las distribuciones a priori sobre el espacio de parámetros de los estimados en los procesos característicos de los modelos propuestos, y como consecuente intervalos de más alta densidad envolviendo de manera significativa las estimaciones. Por lo anteriormente expuesto, se logró encontrar de manera convergente un valor adecuado en estimación para los parámetros.

En lo que atañe a la serie de los rendimientos de NVIDIA el comportamiento de los procesos simulados por MDSM, describió casi de manera exacta la dinámica estocástica de la serie estudiada en comparación al B\&S, el cual cuenta con mayor varianza en los valores simulados y comportamiento platicúrtico.

En la acción de Tesla, los caminos simulados basados en el modelo MDSM mostraron menor volatilidad comparados a la serie de rendimientos originales (serie negra) lo cual indica un comportamiento leptocúrtico en las estimaciones y menor ajuste en yuxtaposición a los modelos estimados para la acción de Apple; este comportamiento se verificó con las distribuciones ilustradas en el cuadro 19, en donde valores simulados por MDSM presentaron un acotamiento más cerrado en los valores de la distribución comparado con el B\&S.

En cuanto a la estimación bayesiana, solamente teniendo en cuenta el comportamiento previo de los parámetros o supuestos frente a la aleatoriedad de estos mismos, se pudo observar una fluctuación sobre las posibilidades en asignación de distribuciones a priori logrando así encontrar valores óptimos para la estimación de los parámetros. En ese sentido, esto quiere decir que no se requiere asignar directamente unos posibles valores para los parámetros, (se requieren menos arranques con el objetivo de encontrar caminos simulados para los procesos); sino más bien, se necesita una asignación aleatoria a ellos por medio de supuestos en términos de distribuciones. Las distribuciones a priori propuestas mostraron como los resultados empíricos obtenidos vía técnicas bayesianas de estimación como el muestreador de Gibbs pueden llegar a ser de gran beneficio para ajustar de manera apropiada modelos en series de tiempo.

En lo que concierne a la comparación entre modelos, MDSM en la literatura clásica suele ser sinónimo de mejor desempeño en el modelamiento de los rendimientos como se comentó anteriormente, ya que, se adapta con los saltos a los distintos cambios del mercado, y se ajusta más a 
la distribución de los rendimientos. En cuanto a la situación bayesiana esta afirmación se logra reafirmar con las simulaciones obtenidas.

A través de los cuadros 10 y 19 se pudo observar el ajuste de las estimaciones sobre el comportamiento de los logaritmos natural de los rendimientos para cada acción, encontrando un grado de ajuste más amplio usando el MDSM. En general se pudo observar cómo MDSM se adapta al comportamiento leptocúrtico y simula el comportamiento de colas pesadas de la distribución de los log rendimientos, característica general presente en este tipo de datos que conforma el mercado de renta variable tecnológico (NASDAQ); de igual manera, se observó el nivel de exactitud de las distintas propuestas prior sobre el MGB en B\&S. Asimismo este último, conserva la ventaja frente a Merton en cuanto a la simplicidad, ya que requiere menos supuestos para la asignación de valores para los parámetros en su forma de estimación estándar y bayesiana.

Finalmente, durante la investigación se logró obtener convergencia en las estimaciones e intervalos de credibilidad validando las propuestas prior para ambos modelos. Estas propuestas fueron reevaluadas posteriormente sobre las distintas cadenas estimadas, presentando al final ningún tipo de problemas relacionados a autocorrelación sobre estas mismas. Una vez estimadas las distribuciones posteriores de los parámetros se pudo evidenciar por un ejercicio de simulación como los caminos aleatorios generados con MDSM se ajustan de mejor manera a la dinámica estocástica de las acciones analizadas.

\section{Referencias}

[1] Castillo, M. (2009). Aplicacion del modelo merton de difusion con saltos para evaluar opciones obre el índice de precios y cotizaciones en un ambiente de alta volatilidad. Escuela Superior de Economía.

[2] Conchi, A. (11 de 2012). Metodos de cadenas de Markov Monte Carlo. Obtenido de http://halweb.uc3m.es/esp/Personal/personas/causin/esp/2012-2013/ SMB/Tema8.pdf.

[3] Fathaleden Asiri, F. (2018). The Price of Stocks, Geometric Brownian Motion, and Black and Scholes Formula. Theses, Dissertations, and Major Papers - University of Windsor - Scholarship at UWindsor.

[4] Gugole, N. (2016). Merton Jump-Diffusion Model Versus The Black and Scholes Approach for the logreturns and volatility smile fitting. International Journal of Pure and Applied Mathematics, 109(3), 719736.

[5] Gupta \& Reisinger. (2012). Robust Calibration of Financial Models Using Bayesian Estimators. Mathematical Institude.

[6] Jones, C. S., Simon, \& Hall Simon, C. (1998). Bayesian Estimation of Continous-Time Finance Models. University of Rochester.

[7] K. K., D. K., \& M. S. (2014). Comparison of Jump-Diffusion Parameters Using Passage Times Estimation. Journal of Applied Mathematics, Volume 2014, Article ID 975418, 5 pages.

[8] Kou, S. (2008). Jump-Diffusion Models for Asset Pricing in Financial Engineering. Handbooks in OR \& MS, Vol 15, 73-116.

[9] Merton, R. (1976). Option Pricing when underlining stock returns are discontinous. Journal of Financial Economics 3, 125-144.

[10] Merton, R., \& Venegas-Martinez, F. (2021). Tendencias y perspectivas de la ciencia financiera: Un artículo de revisión 16(1). Revista Mexicana de Economía y Finanzas, pp 1-15. 
[11] Plummer, M. (28 de June de 2017). Obtenido de JAGS Version 4.3.0 User manual: https://web.sgh.waw.pl/ atoroj/ekonometria_bayesowska/jags_user_manual.pdf

[12] Plummer, M. (6 de Noviembre de 2019). CRAN R. Obtenido de Package 'rjags': https://cran.rproject.org/web/packages/rjags/rjags.pdf

[13] Rifo, L. L., \& Torres, S. (2009). Full Bayesian analysis for a class of jump-diffusion models. Communications in Statistics - Theory and Methods, Volume 38, Issue 8.

[14] Ross, S. (1996). stochastic processes (second edition ed.).

[15] Tang, F. (2018). Merton Jump-Diffusion Modeling of Stock Price Data. Sweeden: Linnæus University Department of mathematics.

[16] Tankov \& Voltchkova. (1992). Jump-diffusion models: a practitioner's guide. Laboratoire de Probabilités et Modèles Aléatoires.

[17] Tunaru \& Zheng. (2017). Parameter Estimation Risk in Asset Pricing and Risk Management: A Bayesian Approach. CEQUFIN.

[18] Venegas-Martínez, F. (2008). Riesgos financieros y económicos: Productos derivados y decisiones económicas bajo incertidumbre. México: Cengage Learning

[19] Zhang, R. (2017). Application of the Merton Jump Diffusion Model in S\&P500. Obtenido de Github: https://ionides.github.io/531w16/final_project/Project06/final_report.html\#global-search-for-themles

\section{Apéndice}

Cuadro A1. Distribución y cadenas en la estimación de $\beta$, B\&S, logaritmo natural de los rendimientos

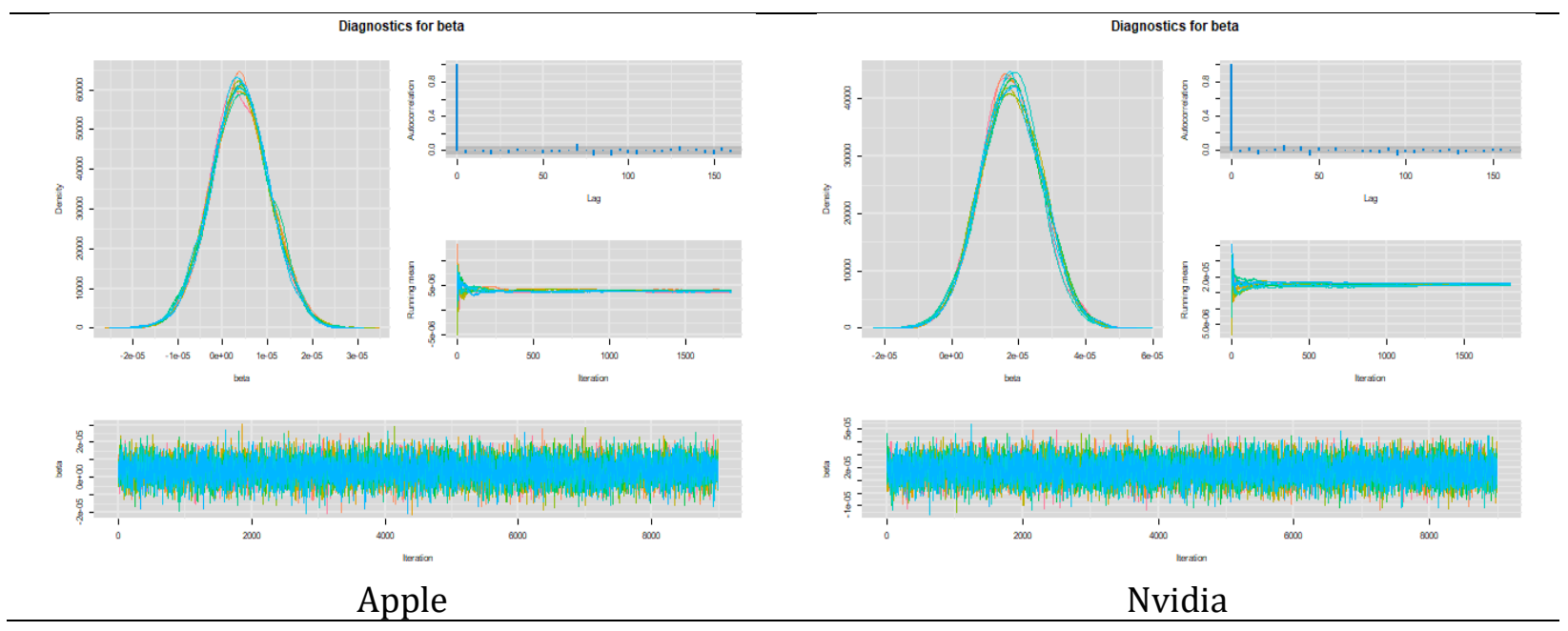




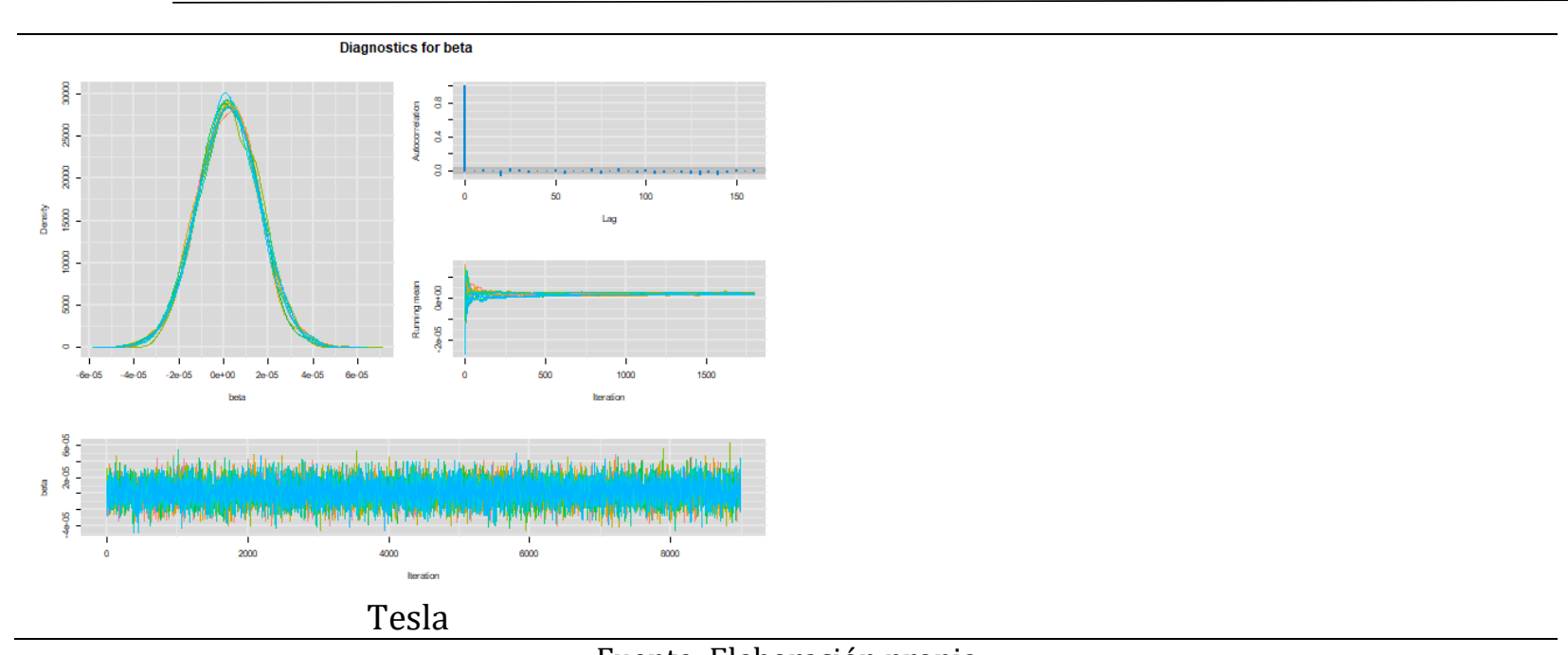

Fuente: Elaboración propia

Cuadro A2. Distribución y cadenas en la estimación de $\sigma^{2}$, B\&S, logaritmo natural de los rendimientos.

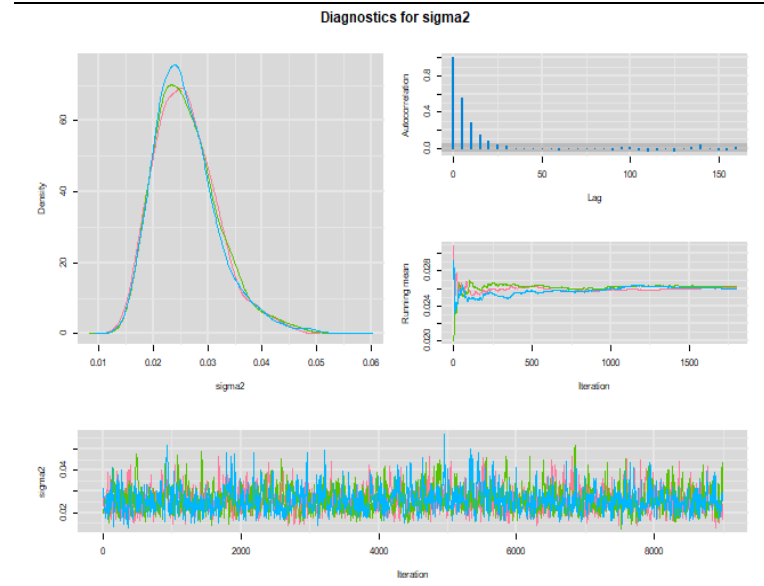

Apple
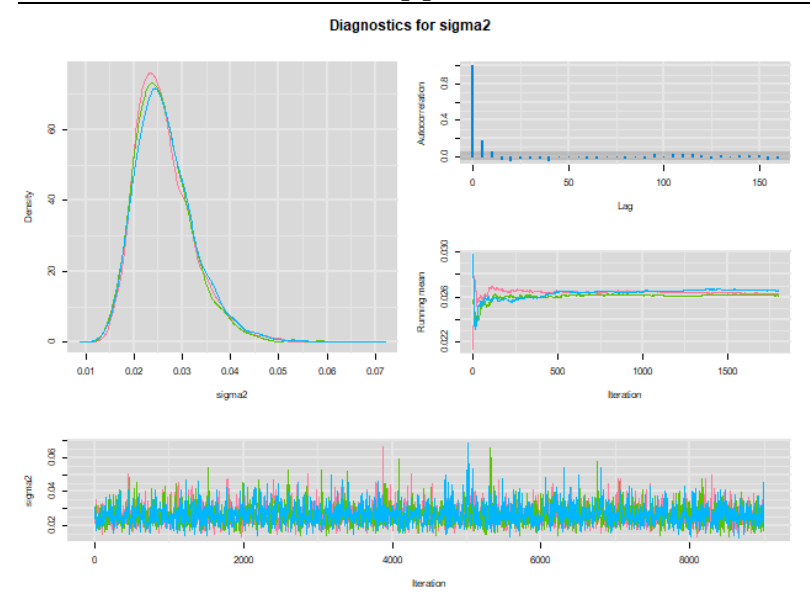

Tesla
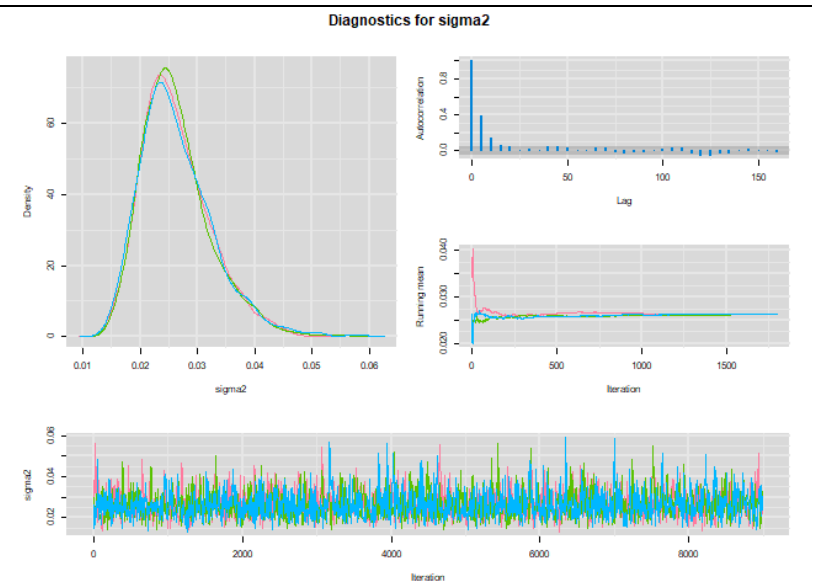

Nvidia 
Cuadro A3. Distribución y cadenas en la estimación para el desvío (deviance), B\&S, logaritmo natural de los rendimientos

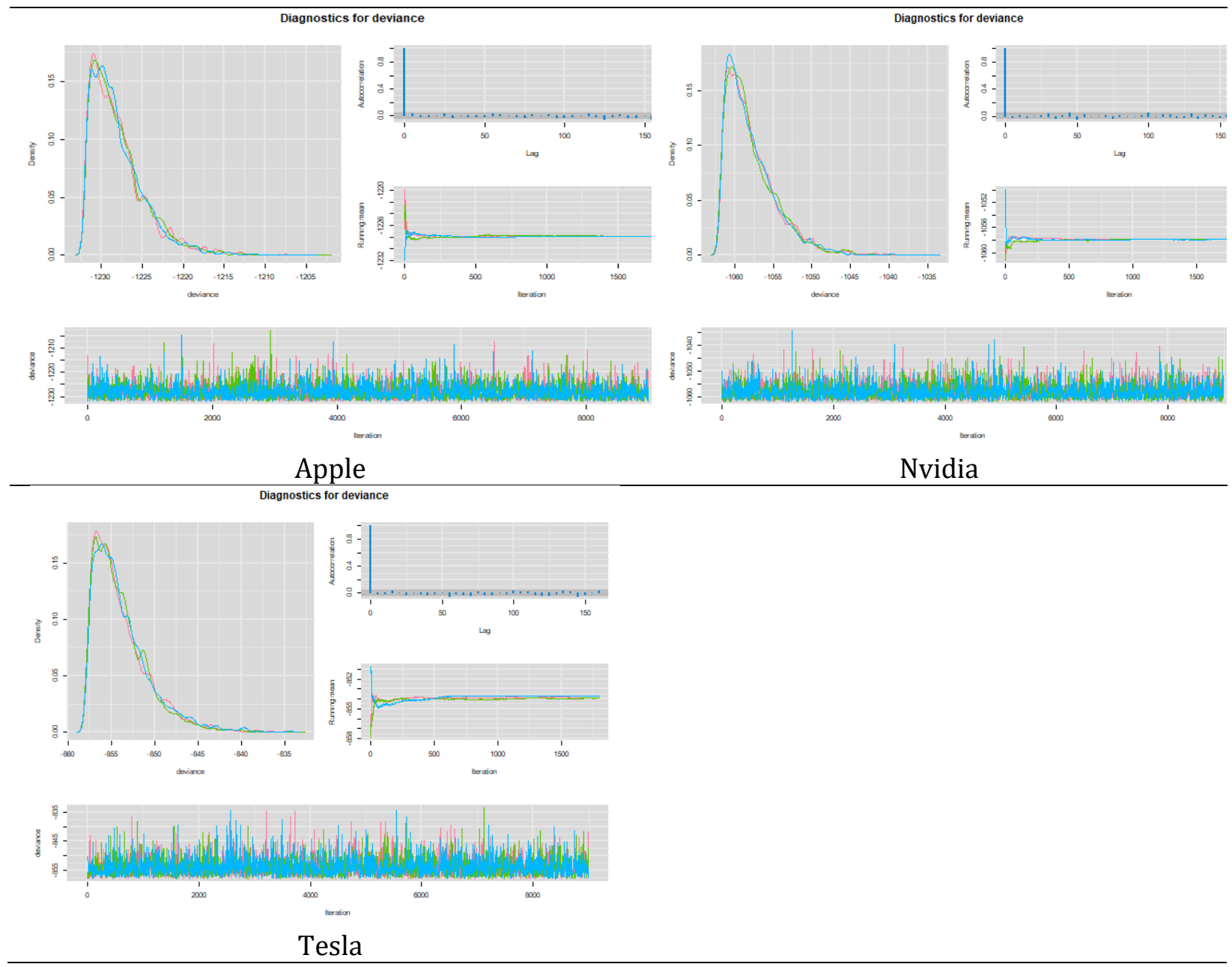

Cuadro A4. Caminos simulados sobre la serie original del logaritmo natural de los rendimientos para las acciones: Apple, Nvidia y Tesla (serie de color negro), usando las estimaciones encontradas de manera bayesiana para B\&S.

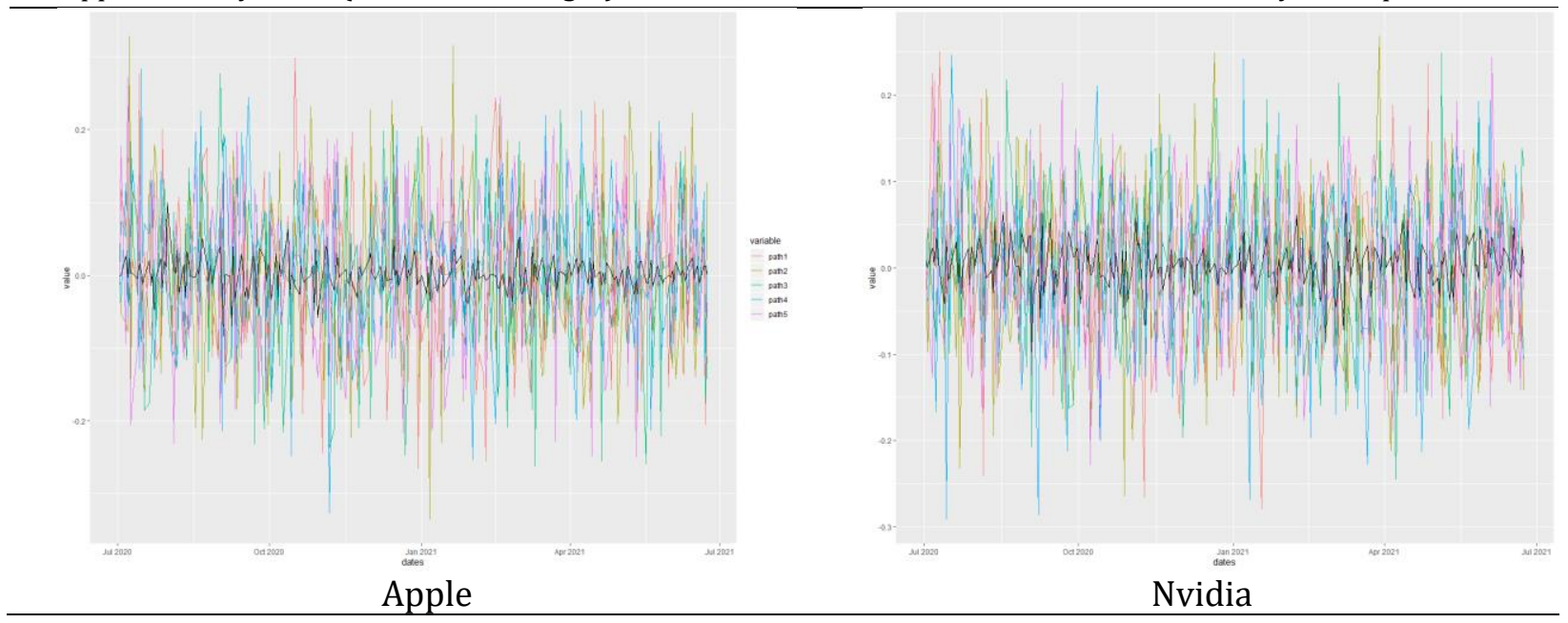




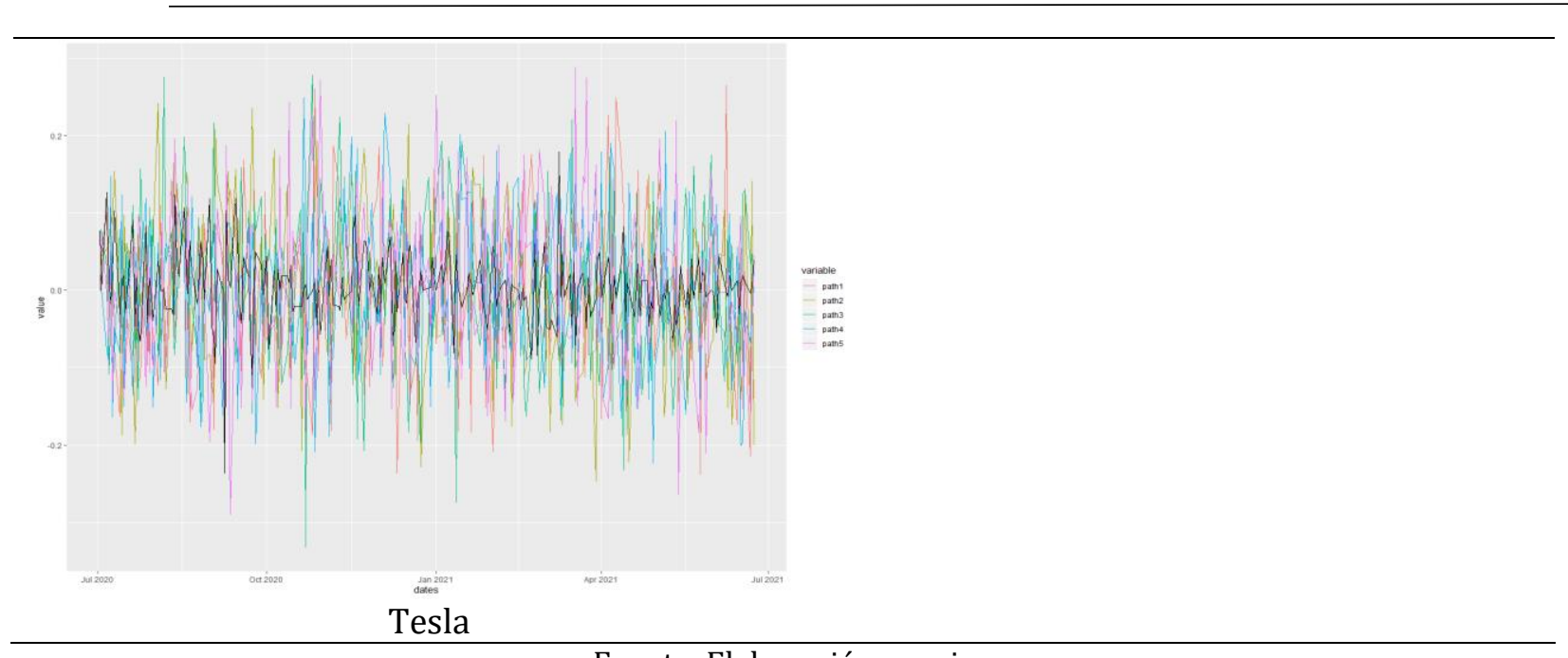

Fuente: Elaboración propia

Cuadro A5. Diagnósticos de convergencia para el parámetro $\delta^{2}$, modelo MDSM, logaritmo natural de los rendimientos.

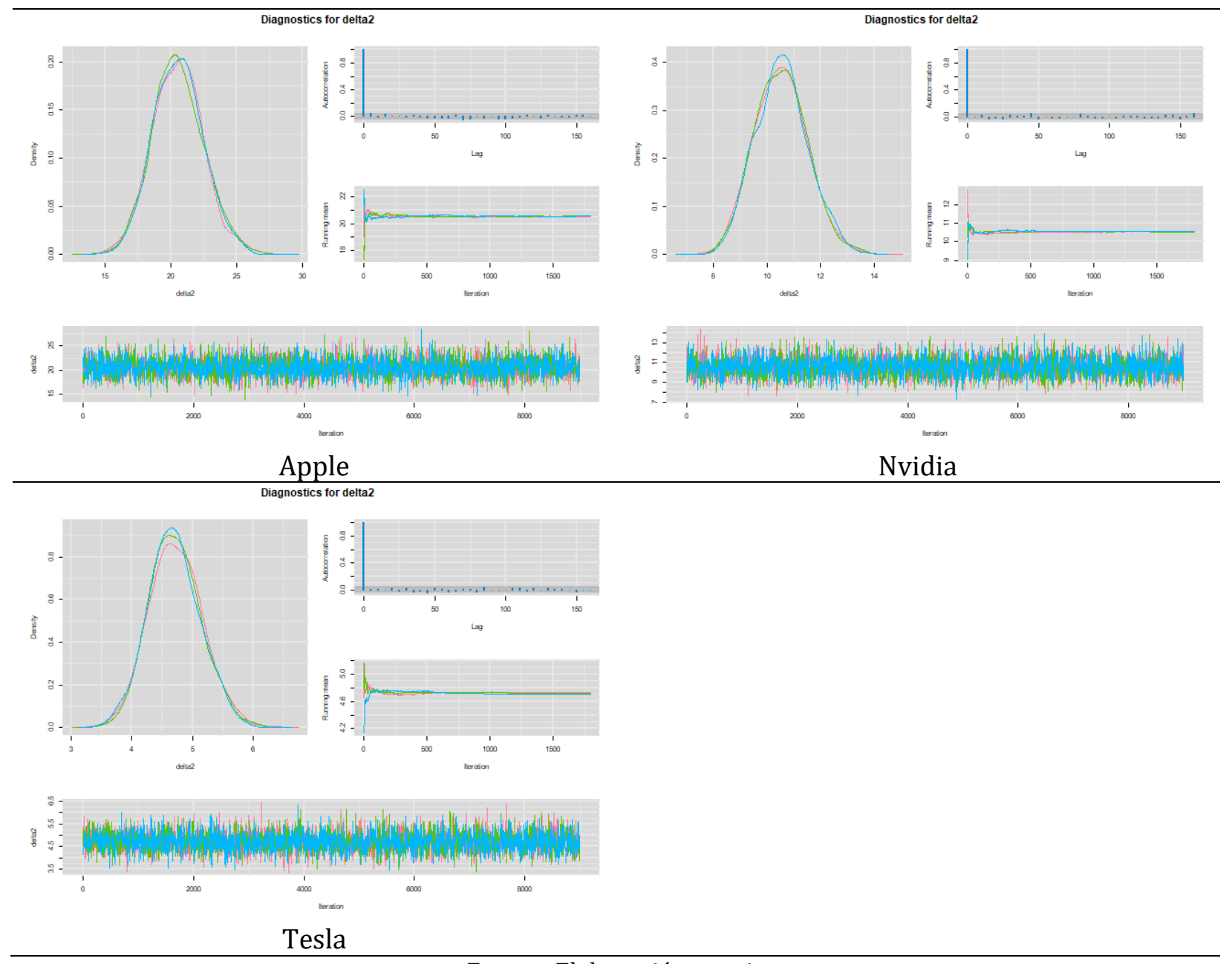

Fuente: Elaboración propia 
REMEF (The Mexican Journal of Economics and Finance)

Estimación bayesiana del modelo de difusión con saltos de Merton

Cuadro A6. Diagnósticos de convergencia para el parámetro $\lambda$, modelo MDSM, logaritmo natural de los rendimientos.
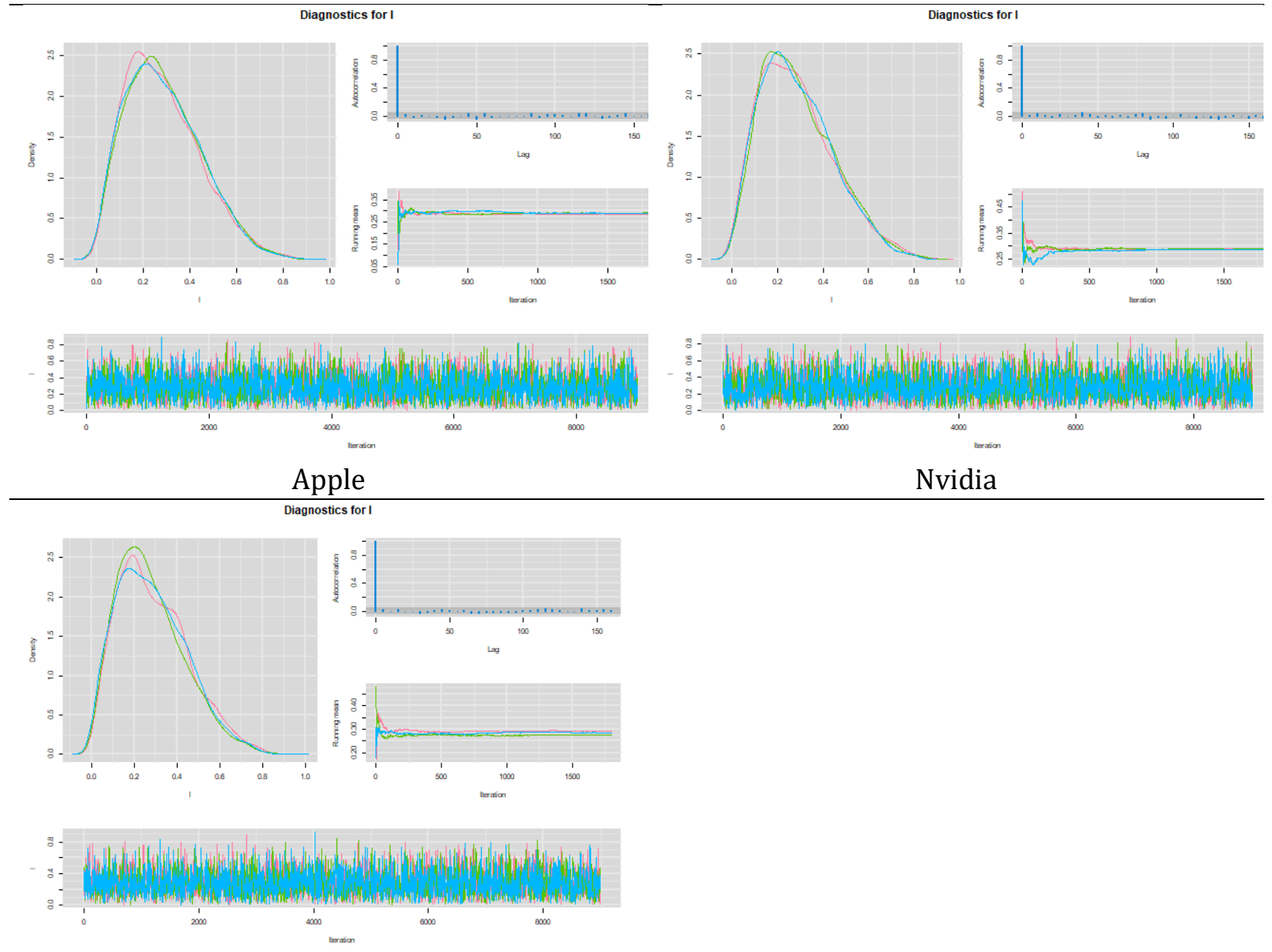

Tesla

Fuente: Elaboración propia

Cuadro A7. Diagnósticos de convergencia para el parámetro $m$, modelo MDSM, logaritmo natural de los rendimientos.

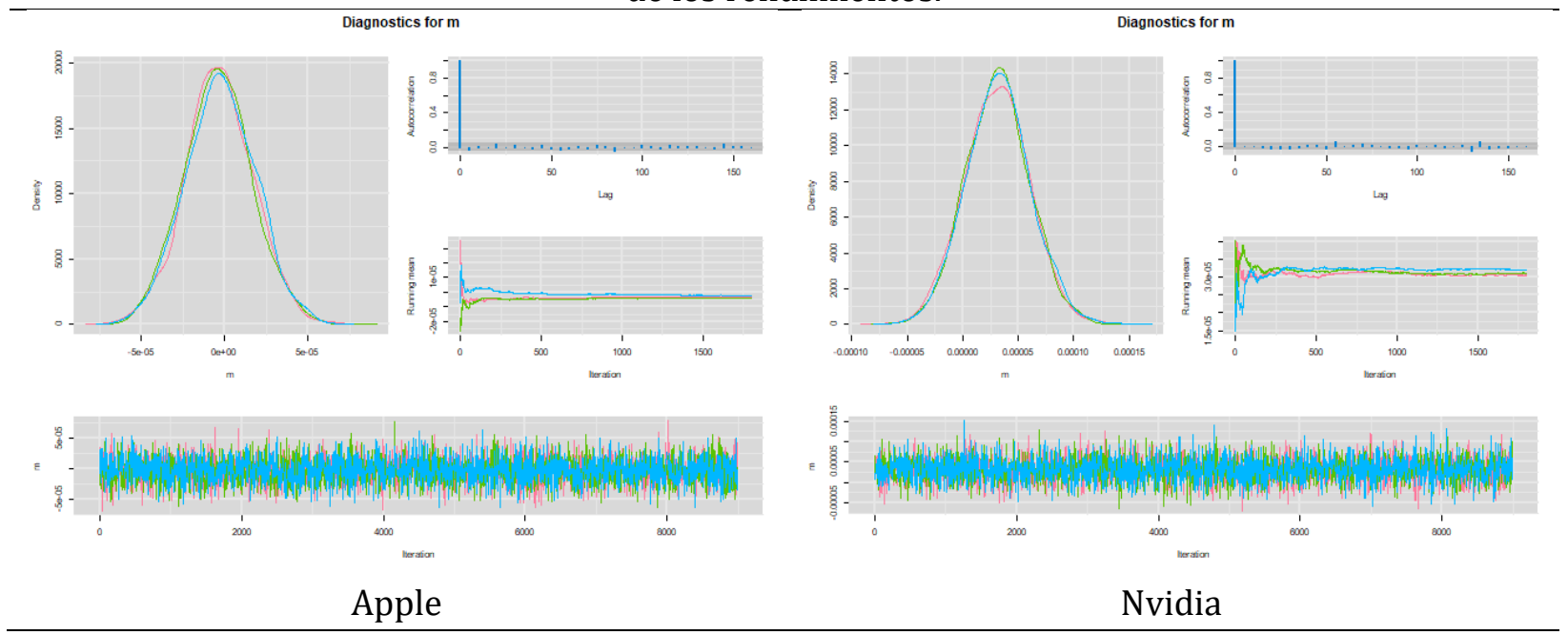




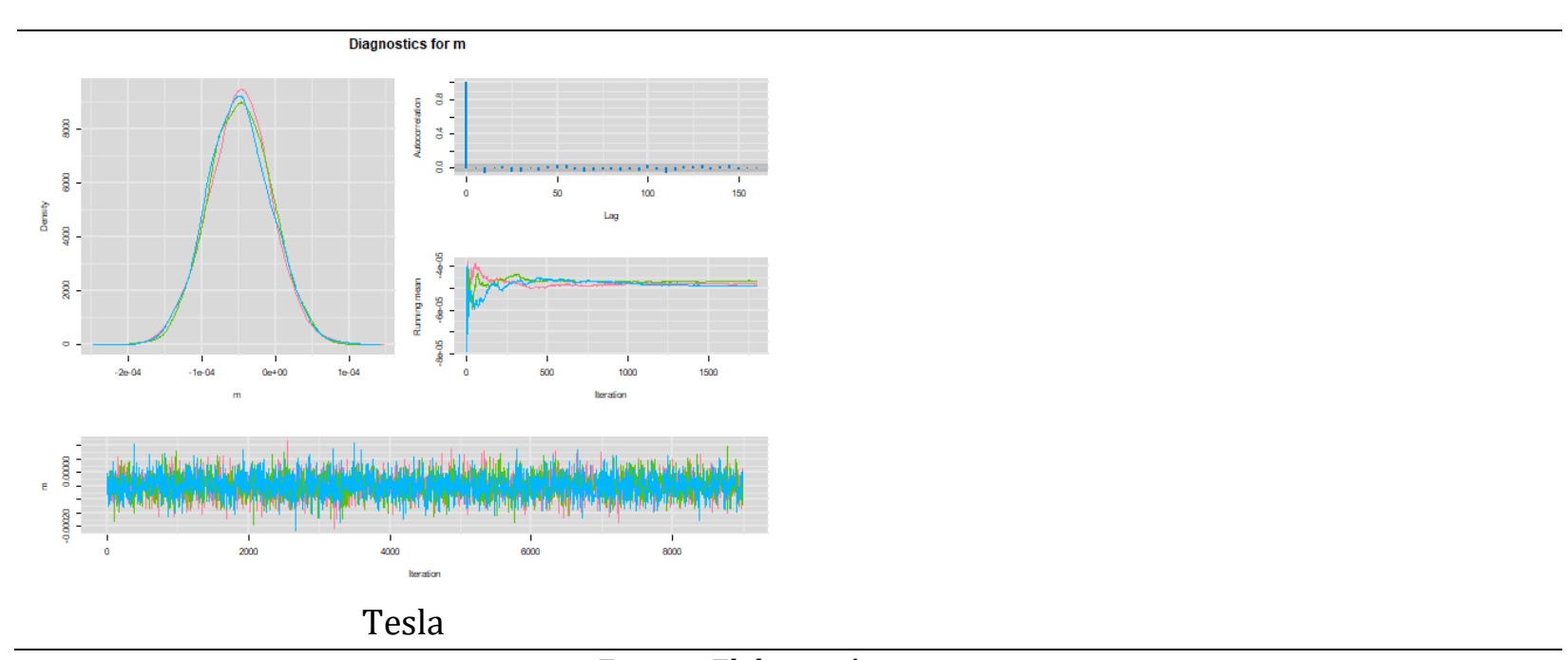

Fuente: Elaboración propia

Cuadro A8. Diagnósticos de convergencia para el parámetro $\mu$, modelo MDSM, logaritmo natural de los rendimientos.
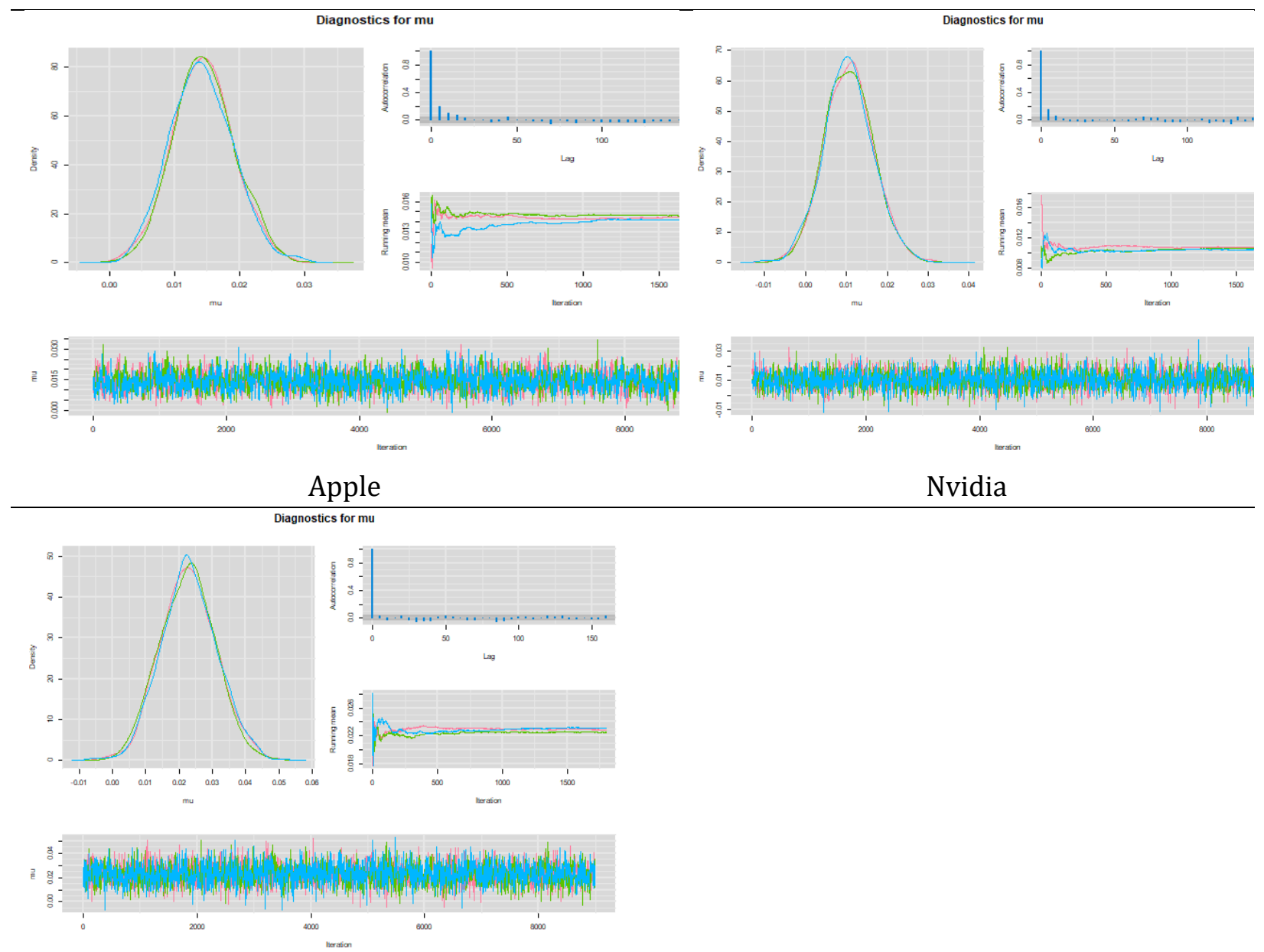

Tesla 
Cuadro A9. Diagnósticos de convergencia para el parámetro $\sigma^{2}$, modelo MDSM, logaritmo natural de los rendimientos.
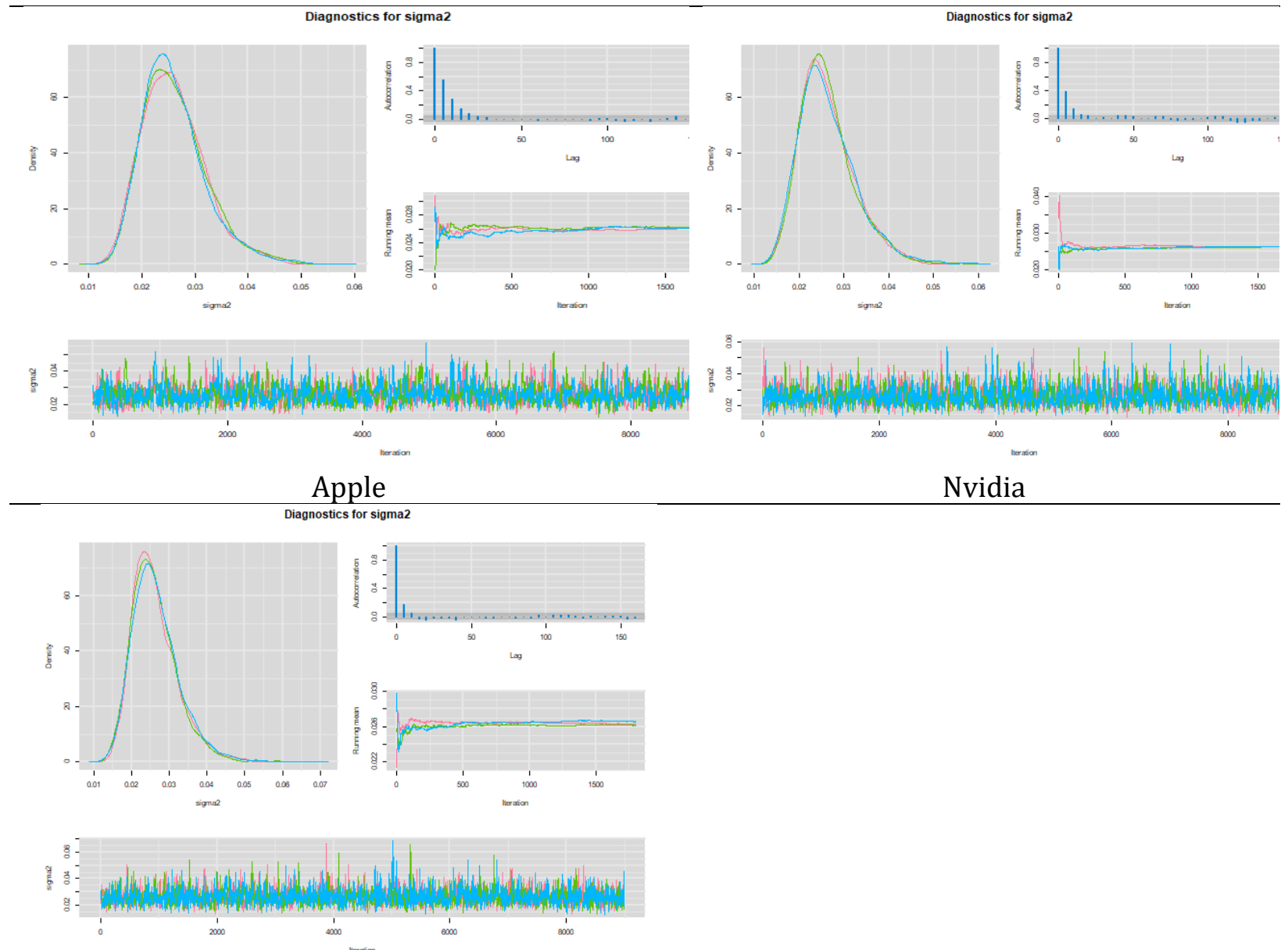

Tesla

Fuente: Elaboración propia

Cuadro A10. Diagnósticos de convergencia para el desvío (deviance), modelo MDSM, logaritmo natural de los rendimientos.

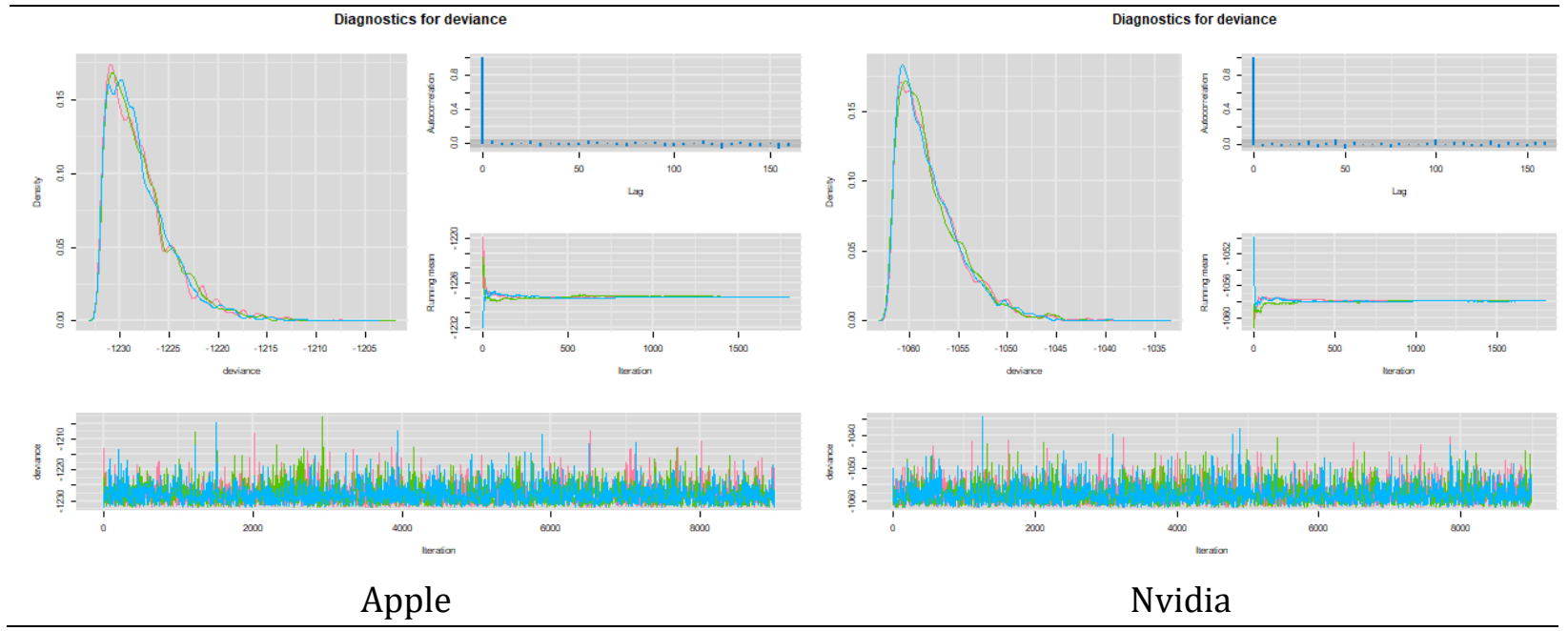




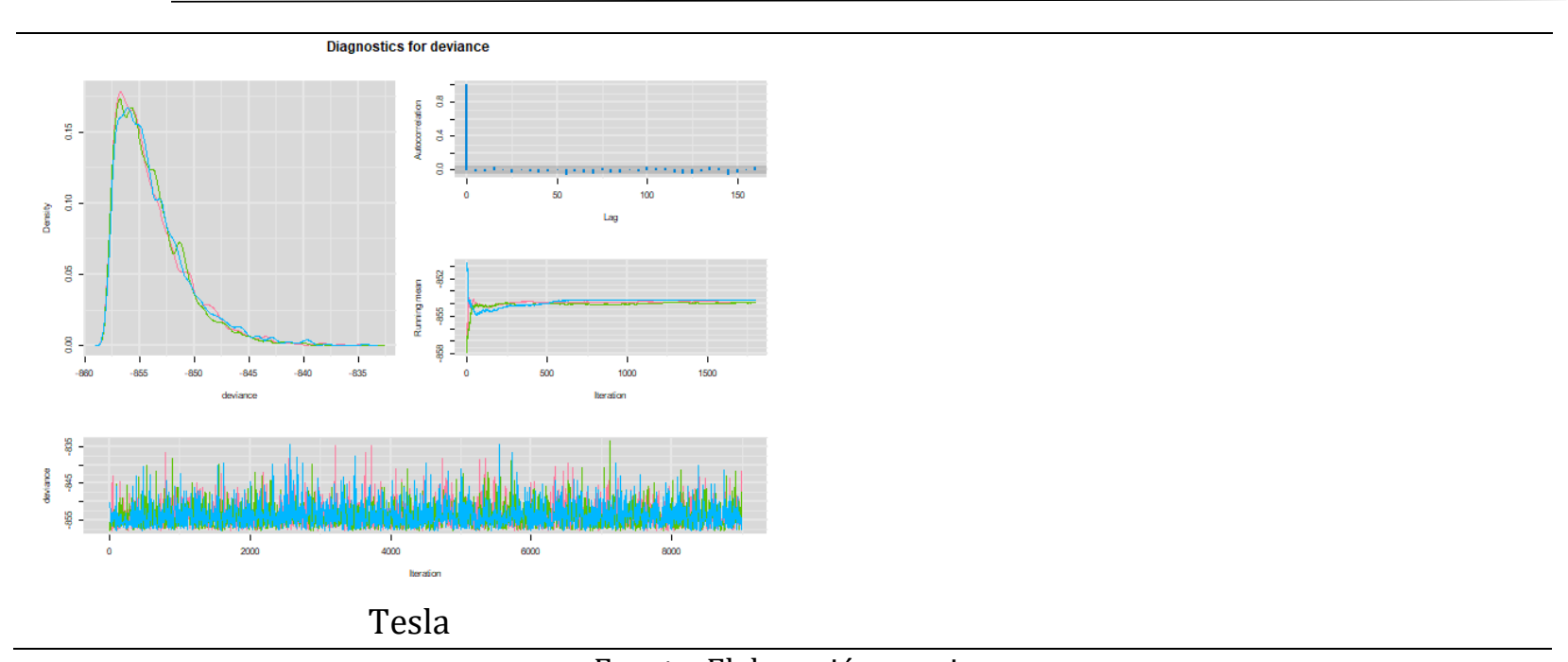

Fuente: Elaboración propia

Cuadro A11. Caminos simulados sobre la serie original del logaritmo natural de los rendimientos para las acciones: Apple, Nvidia y Tesla (serie de color negro), usando las estimaciones encontradas de manera bayesiana para el MDSM

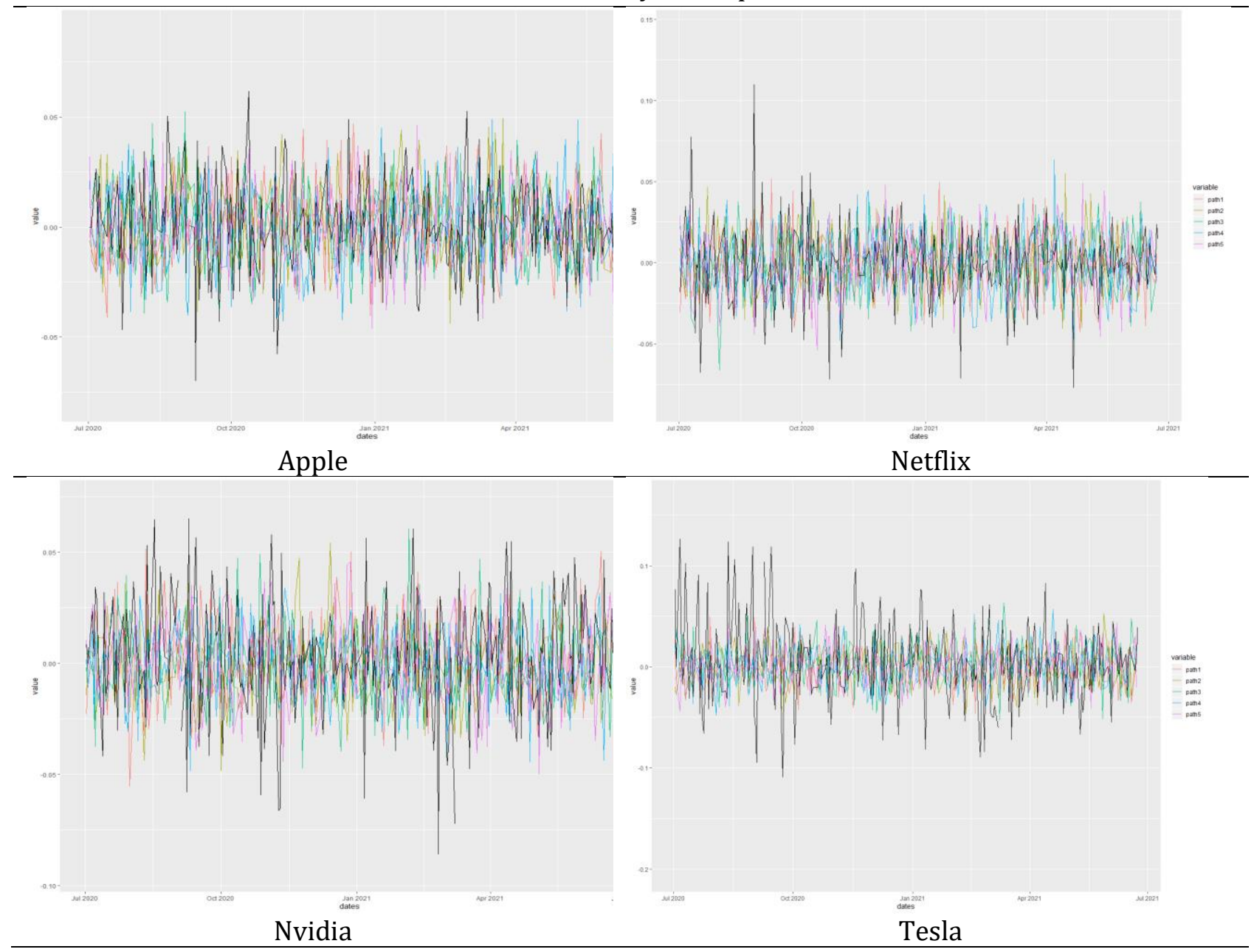

Fuente: Elaboración propia 
Cuadro A12. Distribuciones del logaritmo natural de los rendimientos (Rt) comparados a procesos simulados con el B\&S y MDSM.

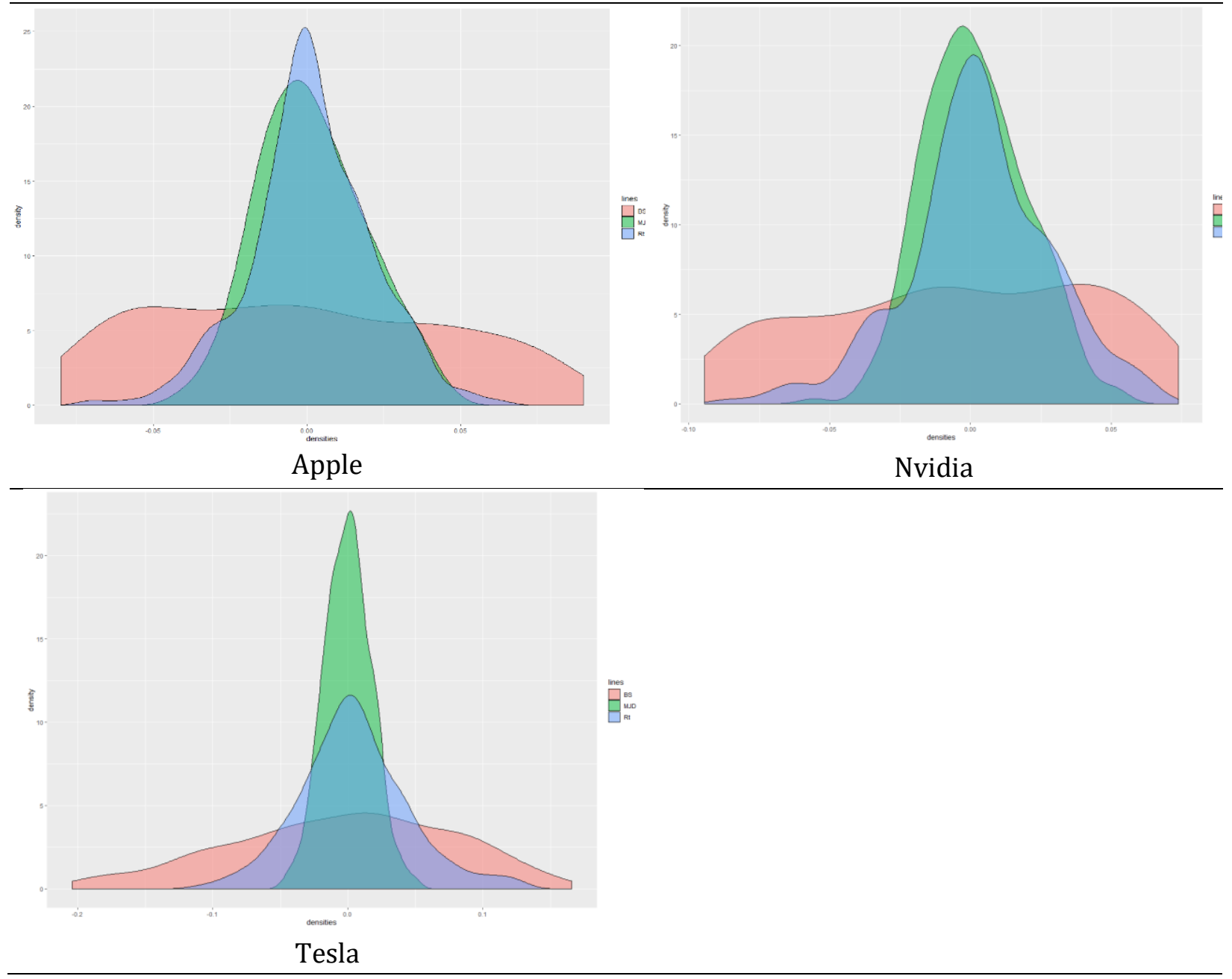

Fuente: Elaboración propia 